\title{
Polyallylamine assisted synthesis of 3D branched AuNPs with plasmon tunability in the vis-NIR region modification.
}

Silvia Nuti, ${ }^{\mathrm{a}, \mathrm{b}, \#}$ Carlos Fernández-Lodeiro, ${ }^{\mathrm{c}, \mathrm{d}, \#}$ Javier Fernández-Lodeiro, ${ }^{* \mathrm{a}, \mathrm{b}}$ Adrián FernándezLodeiro, ${ }^{\mathrm{a}, \mathrm{b}}$ Jorge Pérez-Juste, ${ }^{\mathrm{c}, \mathrm{d}}$ Isabel Pastoriza-Santos, ${ }^{\text {c.d }}$ Alex P. LaGrow, ${ }^{\mathrm{e}}$ Olivier Schraidt, ${ }^{\mathrm{e}}$ José Luis Capelo-Martínez, ${ }^{\mathrm{a}, \mathrm{b}}$ Carlos Lodeiro.*a,b

a BIOSCOPE Group, LAQV@REQUIMTE, Chemistry Department, NOVA School of Science and Technology, NOVA University Lisbon, Caparica Campus, 2829-516 Caparica, Portugal

${ }^{\mathrm{b}}$ PROTEOMASS Scientific Society, Rua dos Inventores, Madam Parque, Caparica Campus, 2829516 Caparica, Portugal

${ }^{\mathrm{c}}$ CINBIO, Universidade de Vigo, Departamento de Química Física, Campus Universitario Lagoas Marcosende, 36310 Vigo, Spain

${ }^{\mathrm{d}}$ Galicia Sur Health Research Institute (IIS Galicia Sur), SERGAS-UVIGO, 36310 Vigo, Spain

${ }^{\mathrm{e}}$ International Iberian Nanotechnology Laboratory, Braga, 4715-330, Portugal

\#These authors contributed equally to this work

Keywords: gold nanoparticles - anisotropic nanoparticles - branched nanoparticles plasmon tunability - polyallylamine

\begin{abstract}
This paper describes the synthesis of highly branched gold nanoparticles through a facile seeded growth approach using poly(allylamine hydrochloride) (PAH) as shape inducing agent. The obtained branched Au NPs present highly tunable optical properties in the VisNIR region from ca. $560 \mathrm{~nm}$ to $1260 \mathrm{~nm}$. We controlled the morphology, and therefore the
\end{abstract}


optical response, of the NPs by either changing the gold salt to seed ratio or the fine-tuning of the solution $\mathrm{pH}$. We proposed that the formation of size-dependent $\mathrm{PAH}-\mathrm{AuCl}_{4}{ }^{-}$ aggregates as demonstrated by dynamic light scattering measurements, together with $\mathrm{pH}-$ dependent gold salt speciation might be responsible for the branched morphology. Advanced electron microscopy techniques demonstrated the polycrystalline nature of the NPs and facilitated a better understanding of branched morphology. Additionally, the refractive index sensitivity estimated by the inflection point of the Localized Surface Plasmon Resonance (LSPR) band can be controlled by tuning the nanoparticle branching. Furthermore, the versatility of the PAH chemistry allowed the easy functionalization of the synthesized NPs.

\section{Introduction}

Since the controlled synthesis of anisotropic gold nanoparticles (AuNPs) became feasible, ${ }^{1}$ this class of AuNPs has been rapidly put to use in many different scientific fields for optical, ${ }^{2,3}$ biomedical, ${ }^{4,5}$ and catalytic ${ }^{6}$ applications. The main asset of anisotropic AuNPs is that the Localized Surface Resonance Plasmon (LSRP) band can be tuned "on-demand" through the tight control of the nanoparticle (NP) shape and size, which give rise to new and exciting physicochemical properties. ${ }^{7}$ For instance, unlike the optical signature of gold spherical NPs, which tends to cover a relatively small wavelength range according to the NP diameter, plasmon resonances of gold nanorods can be controlled in an expanded wavelength range from visible to the near-infrared region of the spectrum, by varying the aspect ratio of the nanoparticle. ${ }^{3}$ Nowadays, alongside nanorods there is an extensive library of anisotropic AuNPs available. Thus, the synthesis and characterization of anisotropic branched or starshaped AuNPs NPs have been the focus of many studies in the last decade. The optical 
properties of branched/star-shaped AuNPs are characterized by an intense LSPR band which can be tuned from 600 to $1200 \mathrm{~nm}$ (mainly as a function of the tip length, thickness and angle, but also the core size) and less intense band in the 500-600 region associated with the AuNP core. $^{3}$ Importantly, branched AuNPs can offer a considerable electromagnetic field enhancement (even superior to $\mathrm{Au}$ nanorods) located at the tips. ${ }^{3}$ These attractive optoelectronic properties have been readily adopted in applications ranging from advanced optical sensors $^{8}$ to nanomedical tools. ${ }^{4,9}$ Branched NPs that offer control over the optical properties in a wide range of wavelengths are mostly obtained through a seed-mediated strategy in the presence of a shape-inducing agent such as surfactants (cetyltrimethylammonium bromide, $\mathrm{CTABr},{ }^{10}$ and zwitterionic lauryl sulfobetaine, $\mathrm{LSB},{ }^{11}$ or Triton $\mathrm{X}^{12,13}$ ), polymers (polyvinylpirrolidone, $\mathrm{PVP}^{14,15}$ ), Good's buffers, ${ }^{16}$ or even silver ions in a surfactant-free solution. ${ }^{17,18}$

Although these branched AuNPs are promising candidates for optical or biomedical applications, post-functionalization steps are essential to impart stability as well as functionality to the as-synthesized NP. ${ }^{19,20}$ Unfortunately, the gold surfaces located at the tips possess a high density of low-coordinated atoms being a high surface energy, which could promote the reshaping of the NPs upon surface functionalization, thus altering their optical properties..$^{20}$ In order to prevent any reshaping process, surface modification with different thiol derivatives has been proposed. ${ }^{19}$

The development of novel synthetic routes for the synthesis of branched AuNPs with tunable optical properties using functionalizable shape-inducing agents has been identified as a challenging approach. For instance, polyallylamine hydrochloride (PAH), a positively charged polyelectrolyte with a high degree of primary amine functions and excellent hydrophilic properties, has shown potential as a shape-directing agent in the synthesis of 
anisotropic noble metal NPs (such as platinum or copper), ${ }^{21,22}$ or as a stabilizing and reducing precursor in the synthesis of small spherical AuNPs. ${ }^{23,24}$ Additionally, Tang and coworkers reported a one-pot synthesis of branched AuNPs using PAH as a shape-inducing and capping agent after reduction with ascorbic acid (AA). ${ }^{25}$ The obtained dendritic structures, named nanocorals, showed excellent electrocatalytic activity in ethanol oxidation, but the optical properties were not reported. Interestingly, the amine functionalities of the PAH allow its post-functionalization through either electrostatic interaction ${ }^{26,27}$ or carbodiimide chemistry. ${ }^{27,28}$

Taking into consideration these previous works, we developed a seed-mediated approach for the synthesis of branched AuNPs assisted by PAH. The strategy relies on the prevention of nucleation events while promoting the growth process through the presence of small Au seeds. The careful control of the experimental conditions allows obtaining branched AuNPs with highly tunable optical properties (ca. 560-1260 nm). High-resolution transmission electron microscopy (HR-TEM) in combination with scanning transmission electron microscopy (STEM) tomography reveals a polycrystalline structure and an intricate morphology. Furthermore, the refractive index sensitivity estimated by the inflection point of the Localized Surface Plasmon resonance band can be easily controlled by tuning the nanoparticle branching. Additionally, taking advantage of the surface-exposed amine groups, the branched AuNPs could be easily functionalized with polyacrylic acid-co-maleic acid (PAcMA) via carbodiimide chemistry without affecting their optical properties.

\section{Experimental. Results and discussion}


Polyallylamine hydrochloride (PAH) is a cationic polyelectrolyte with a weak-base character. Recently, Tang et al. reported the one-pot synthesis of branched Au nanocorals where the polyallylamine hydrochloride (PAH) acted as capping and shape control agent and ascorbic acid as reducing agent. ${ }^{25}$ Nevertheless, the optical properties of the so-called $\mathrm{Au}$ nanocoral were not analyzed and no attempts to control the particle size or to study the influence of the $\mathrm{pH}$ were performed. The latter is especially interesting considering that the redox potential of ascorbic acid, Au salt speciation and the degree of ionization in PAH molecules are $\mathrm{pH}$-dependent, ${ }^{29-31}$ which could affect the formation kinetics of $\mathrm{Au}$ nanoparticles as well as the interaction of PAH through the amine groups with the gold salt and the surface of AuNP. ${ }^{32}$

Here, we employed a seeded-growth methodology to fully understand the key parameters affecting the synthesis of branched Au NPs and aiming their size and shape control. ${ }^{33}$ Thus, citrate-stabilized gold nanoparticles with an average size of 3-5 nm, synthesized via the fast reduction with $\mathrm{NaBH}_{4}$ (see Figure $\mathbf{S 1}$ in the SI) ${ }^{34}$ were used as initial seeds. Considering the $\mathrm{pH}$-dependent redox potential of ascorbic acid as well as the ionization degree of $\mathrm{PAH}$, we studied the influence of the $\mathrm{pH}$ on the overgrowth of the Au seeds. The nanoparticle growth was investigated at different $\mathrm{pHs}$, ranging from 2.5 to 7.3 while keeping constant the number of seeds (53.3 pM in terms of NPs concentration), gold salt precursor and ascorbic acid. The Vis-NIR analysis of the resulting colloids revealed that at pHs ranging from 2.5 and 5 the total reduction of the Au salt occurs while $\mathrm{pH}$ above 5 inhibited the reduction of the $\mathrm{Au}$ precursor and even compromised the colloidal stability (Figure S2 in SI). Moreover, in the $\mathrm{pH}$ range $2.5-5$, the optical properties of the resulting nanoparticles are strongly influenced by the $\mathrm{pH}$ and, as expected, this difference in the optical properties is reflected in the morphology of the particles (Figure 1A). While at pH 2.5 the resulting Au colloids show a 
relatively narrow localized surface plasmon resonance (LSPR) band at $c a .557 \mathrm{~nm}$ being the particles fairly spherical (Figure 1B), the gradual increase of $\mathrm{pH}$ from 3.9 to 4.8 gives rise to a red-shift of the LSPR as well as the appearance of a new and less intense plasmon mode at lower wavelengths. Thus at $\mathrm{pH} 4.1$, the AuNPs resemble a popcorn with a core of about 50 $\mathrm{nm}$ and branch lengths of 10-15 nm (Figure 1B,C). Significantly, as the $\mathrm{pH}$ increases to 4.8 the length of these branches increased while the core slightly decreased (Figure 1C-D). This is in agreement with previous reports where the two dipolar resonances were assigned to LSPR modes localized at either the central core of the NPs (located around ca. $520 \mathrm{~nm}$ ) or the tips/branches of the NPs (being its position depending on its length and sharpness). ${ }^{8}$ On the other hand, The highly positive surface potential $(\zeta=+46.1 \mathrm{mV})$ reveals that the resulting NPs are stabilized by PAH. 

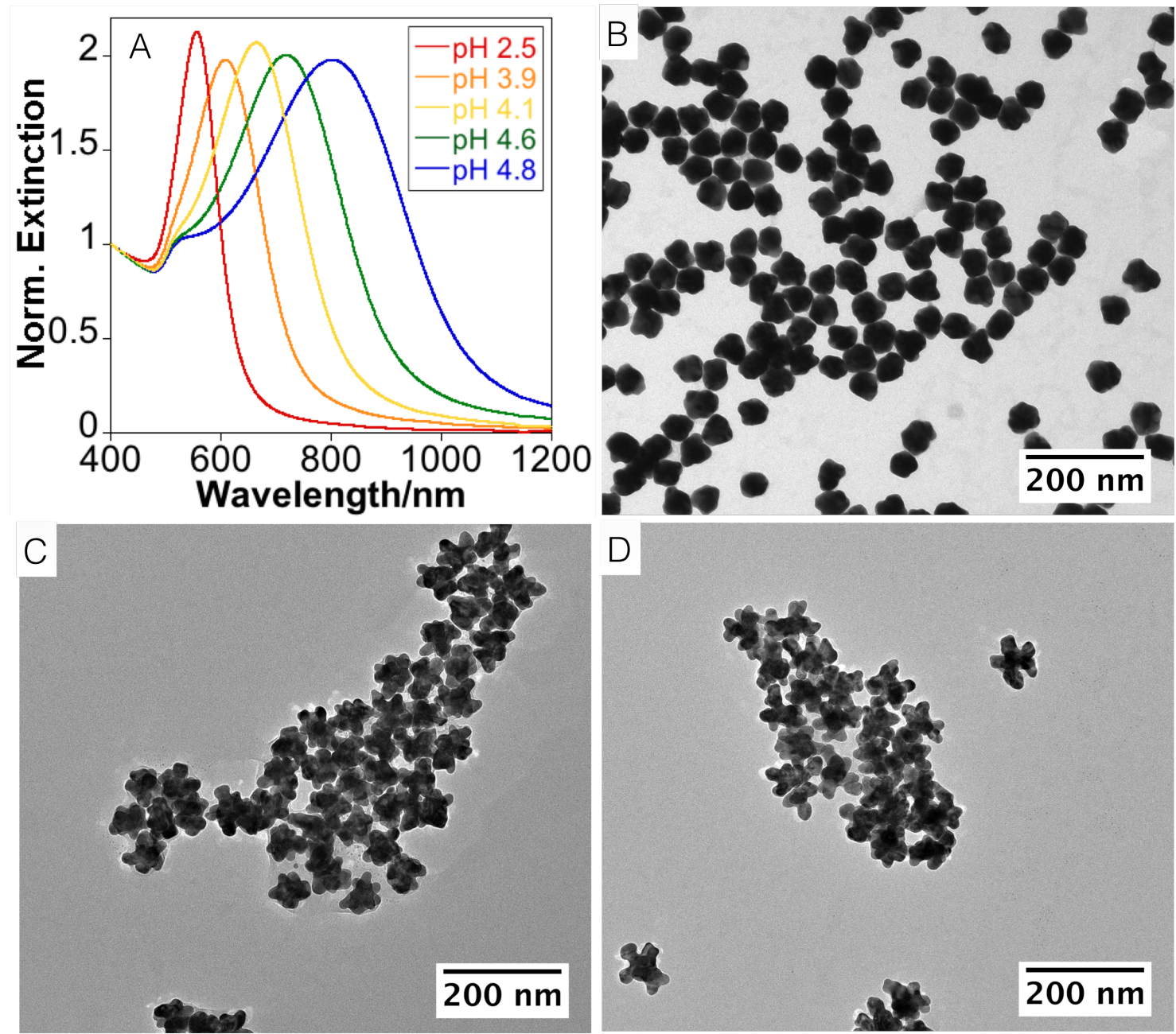

Figure 1. (A) Vis-NIR spectra of the AuNPs synthesized in the presence of PAH at different pHs (2.5, 3.9, 4.1, 4.6 and 4.8) upon injection of gold seeds (final NP concentration: $53.3 \mathrm{pM}$ ). (B-D) Representative TEM images of AuNPs obtained at pH 2.5 (B), 4.1 (C) and 4.8 (D).

Next, we evaluated the influence of the amount of seed on the growth process. Figure $\mathbf{2}$ shows the vis-NIR spectra of the AuNPs obtained at $\mathrm{pH} 4.1$ and varying the amount of seed added from 106.6 pM to $8.9 \mathrm{pM}$. As shown in Figure 2A, as the amount of seeds decreases the LSPR band of the resulting nanoparticles red-shifts evidencing a second band at lower wavelengths, in such a way that it can be fine-tuned from $c a .560 \mathrm{~nm}$ to $1260 \mathrm{~nm}$. These differences in the optical response are reflected in their morphology (Figures $\mathbf{2}$ and S3-S7 in 
SI). For the highest amount of seeds (106.6 pM), the particles are fairly monodisperse with a bumpy morphology and an average size of $49 \pm 6 \mathrm{~nm}$ (Figures 2B and S3). As the amount of seed decreased from $106.6 \mathrm{pM}$ to $8.9 \mathrm{pM}$, the initial bumps evolved into larger branches (Figures 2D-F and S3-S7 in the SI) producing a red-shift of the main LSPR. This evolution in the morphology of the AuNPs is also accompanied by an increase in the mean overall size from $49 \mathrm{~nm}$ to $151 \mathrm{~nm}$ as determined by TEM analysis. Considering the remarkable effect of the solution $\mathrm{pH}$ in the morphology of the Au nanoparticles obtained with the addition of a constant amount of seed (53.31 pM, Figure 1), the study was extended to other seeds amounts. The results obtained with $26.7 \mathrm{pM}$ and $8.9 \mathrm{pM}$ at $\mathrm{pHs} 2.5$ and 4.1 reveal the strong effect of the $\mathrm{pH}$ in the growth of NPs regardless of the amount of seed added (see Figures S8 and $\mathbf{S 9}$ in the supporting information).
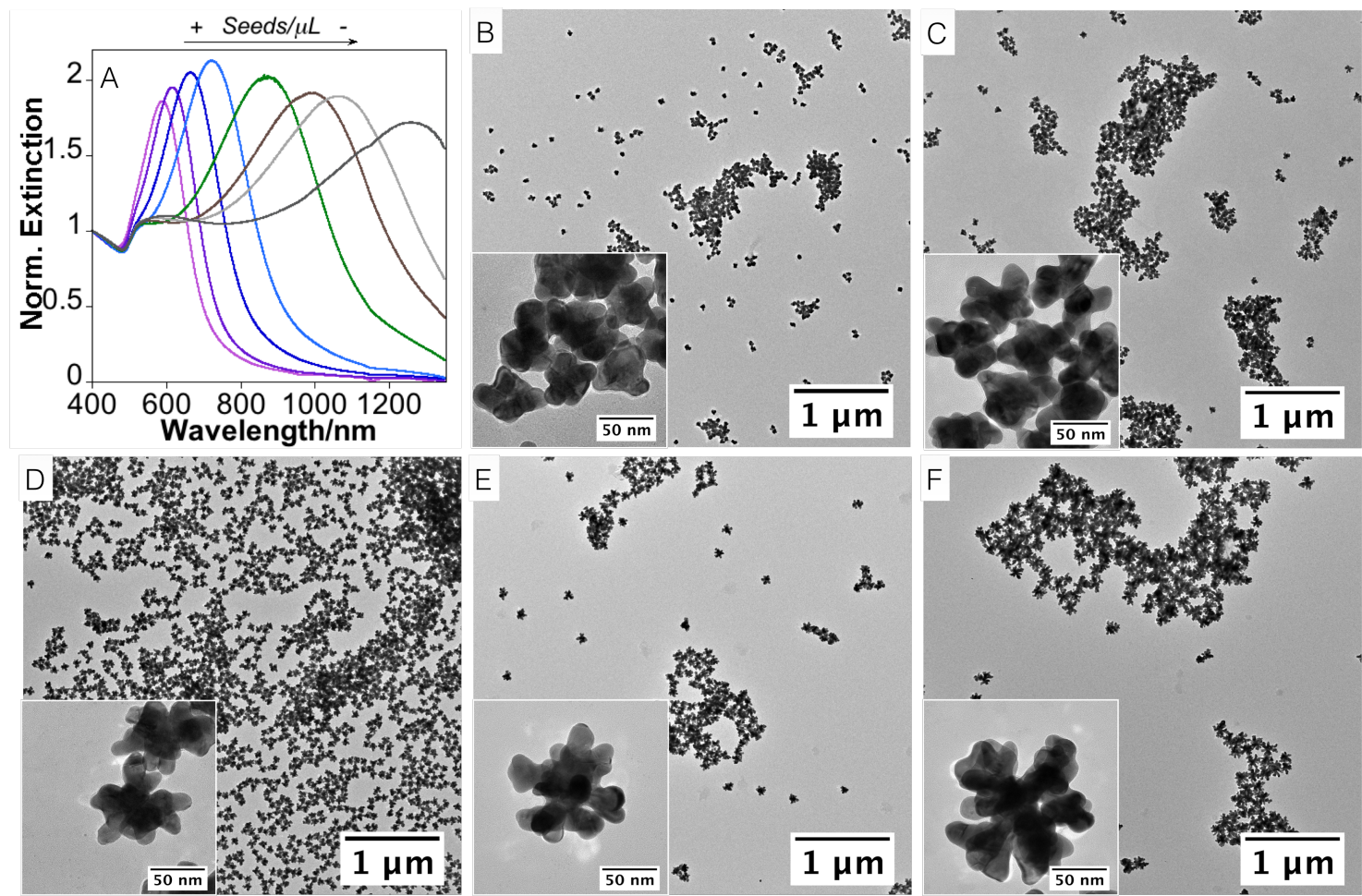

Figure 2. (A) Normalized Vis-NIR spectra of branched AuNPs obtained using different amounts of Au seeds.

The final Au NPs concentrations were: 8.9 pM, 13.3 pM, 17.8 pM, 26.7 pM, 35.4 pM, 53.3 pM, 71.1 pM, 106.6 
pM. (B-F) Representative TEM images of AuNPs obtained using 106.6 pM (B), 53.3 pM (C), 35.4 pM (D), 26.7 $p M(E)$ and $8.9 p M(F)$ of Au seeds.

To investigate the NPs formation, the time evolution of the optical properties during the AuNP growth (for $53.3 \mathrm{pM} \mathrm{Au}$ seeds) was analyzed at $\mathrm{pH} 2.5$ and 4.1. At $\mathrm{pH} 2.5$ the reaction is complete in less than 3 minutes since the well-defined LSPR centered at $c a .555$ $\mathrm{nm}(\mathrm{t}=3 \mathrm{~min})$ did not undergo noticeable changes at longer times (see Figure S10A). On the other hand, at $\mathrm{pH} 4.1$ (see Figure S10B) the kinetic study revealed that the reaction proceeds a bit slower than at 2.5 and two different stages could be distinguished. Thus, there is a fast initial stage where the reduction of the gold salt takes place in five minutes, monitored through the extinction at $400 \mathrm{~nm},{ }^{35}$ giving rise to Au nanoparticles with a broad and poorly defined LSRP band (see red spectrum in Figure S10B). Besides there is a second stage, from 5 minutes to ca. 240 minutes, where no further reduction of gold salt is produced and the morphological evolution of the nanoparticles takes place as indicated by the blue-shift, sharpening and increase in the intensity of the main LSPR band (see Figure S10B). It should be pointed out that these optical changes were also observed for Au NPs growth from 26.7 pM Au seeds (see Figure S10C in the SI).

To investigate more in-depth the particles growth, aliquots were withdrawn at different reaction times during the formation of Au NPs and analyzed by TEM and Vis-NIR spectroscopy after inhibiting the growth process via the addition of thiolated PEG (Figure 3A and Figure S11 in the SI). As shown in Figure 3A and Figure S11 for $53.3 \mathrm{pM} \mathrm{Au}$ seeds and $\mathrm{pH} 4.1$, the gold salt reduction is very fast, thus $\sim 5$ seconds after the seed addition close to $70 \%$ of the gold salt is reduced (estimated from the extinction at $400 \mathrm{~nm}$ ) and the resulting NPs exhibit an LSPR band located at $c a .610 \mathrm{~nm} .{ }^{35,36}$ TEM analysis revealed that 
these particles present small and multiple bumps at their surface and an overall average diameter of $c a .55 \mathrm{~nm}$ (Figure 3B and S12). From 15 seconds to 5 minutes the initial bumps grow in random directions leading to an increase in the number and length of the branches (see Figure 3C and Figure S13A-D in the SI). Eventually, after 5 minutes the AuNPs gradually evolve into a morphology with a lower degree of branching but accompanied by an increase in its curvature, as indicated by the narrowing an increase in the intensity of the main LSPR (Figure 3D and Figure S13E-H).
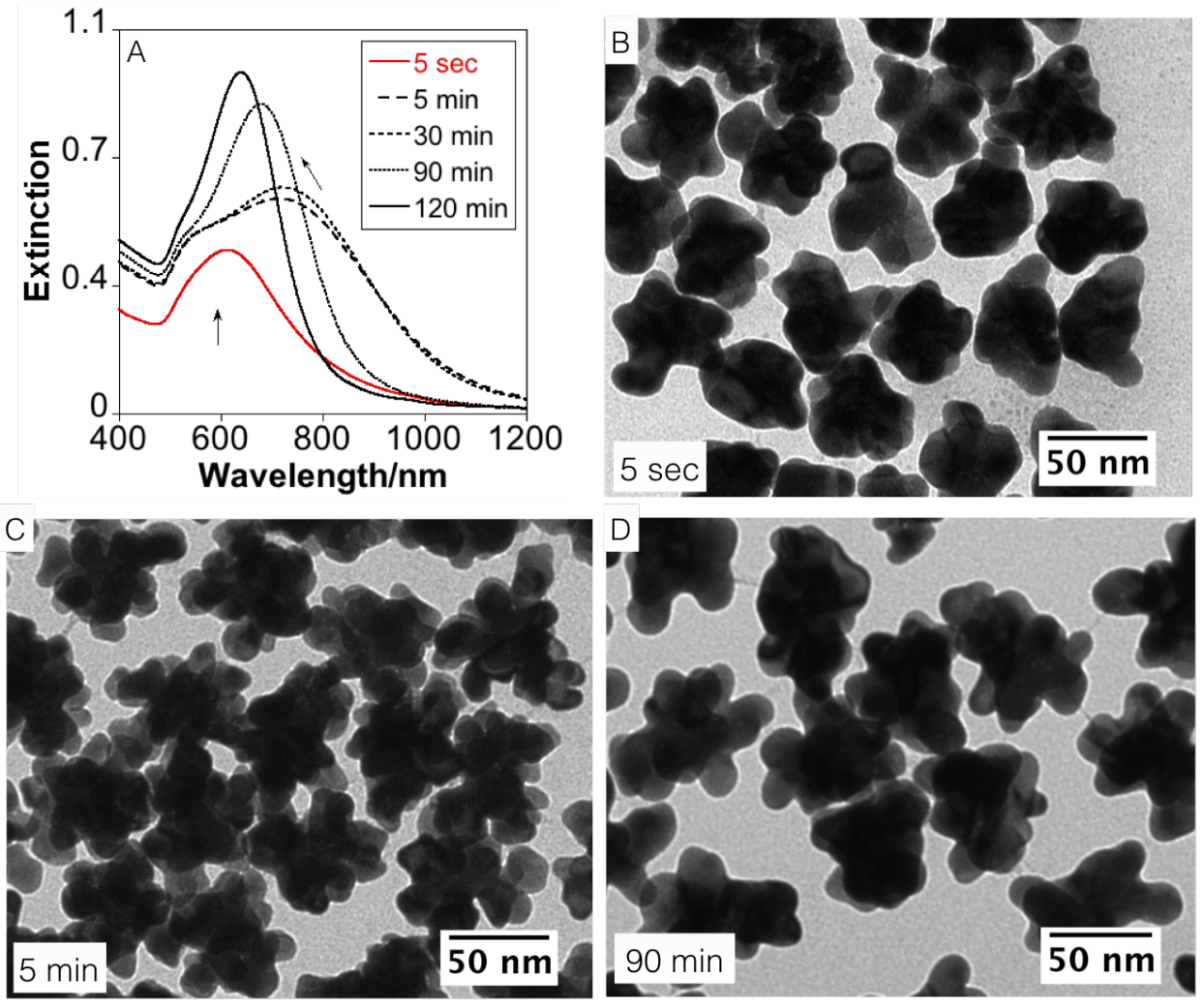

Figure 3: (A) Time-resolved vis-NIR spectra of the AuNP growth at pH 4.1 upon the addition of obtained Au seeds (53.3 pM in NP concentration). (B-D) Representative TEM images of the Au intermediates after arresting the growth process at the indicated times. 
The morphology and structure of branched AuNPs obtained with $53.3 \mathrm{pM}$ and $8.9 \mathrm{pM}$ gold seeds were further investigated by high-resolution TEM (HRTEM) and high-angle annular dark-field scanning transmission electron microscopy (HAADF-STEM), Figure 4. The analysis of the AuNPs reveals that the branches show different crystallographic orientations with twin boundaries between them (see black arrows in Figure 4D and 4J). Interestingly, the HAADF analysis reveals small regions with slightly different contrast mostly seen near lattice defects (see red arrows in Figure 4B,C,H and I), which could indicate the presence of holes in the internal structure of the NP. Besides, we performed 3D structural analysis through electron tomography to obtain further information regarding the morphology as well as to confirm the presence of holes in its structure. Figure 5 and S14 shows representative electron tomography reconstruction of different AuNPs obtained at $\mathrm{pH} 4.1$ using $53.3 \mathrm{pM}$ or $8.9 \mathrm{pM}$ Au seeds, where their multiple branched morphology is confirmed. Additionally, the orthoslices through the reconstructions show the presence of holes within the structure (Figure 5C and D and S14), which are more evident in the larger particles (Figure S14). Animated visualizations of the obtained reconstructions are provided as Supporting Information for both types of AuNPs (see Movies S1 to S4 in the Supporting Information). 

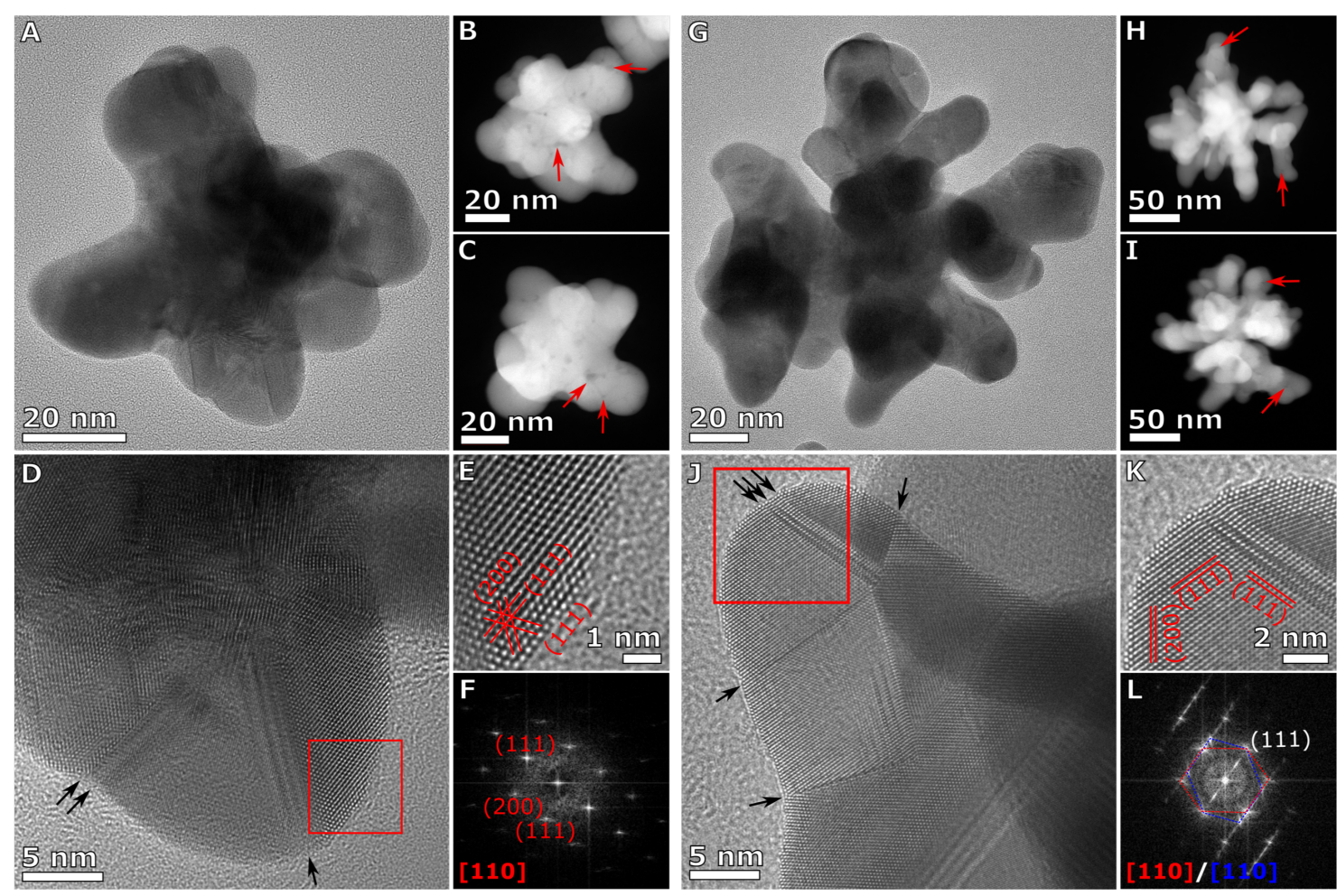

Figure 4. (A, G) HRTEM images of AuNPs obtained with $53.3 \mathrm{pM}(A)$ and $8.9 \mathrm{pM}(\mathrm{G})$ gold seeds. (B-C, H-I) HAADF-STEM images of individual particles obtained with $53.3 \mathrm{pM}(B-C)$ and $8.9 \mathrm{pM}(\mathrm{H}-\mathrm{I})$ gold seeds. The red arrows indicate holes in the structure. (D, J) HRTEM images of a branch of the AuNP from $A(D)$ and $G$ (J). The black arrows indicate the twin planes. (E, K) HRTEM images of the highlighted region shown in D (E) and $J(K)$ with the lattice spacing in the [110] zone axis indicated by red lines. $(F, L)$ FFT patterns obtained from $D(F)$ and $J(L)$. 

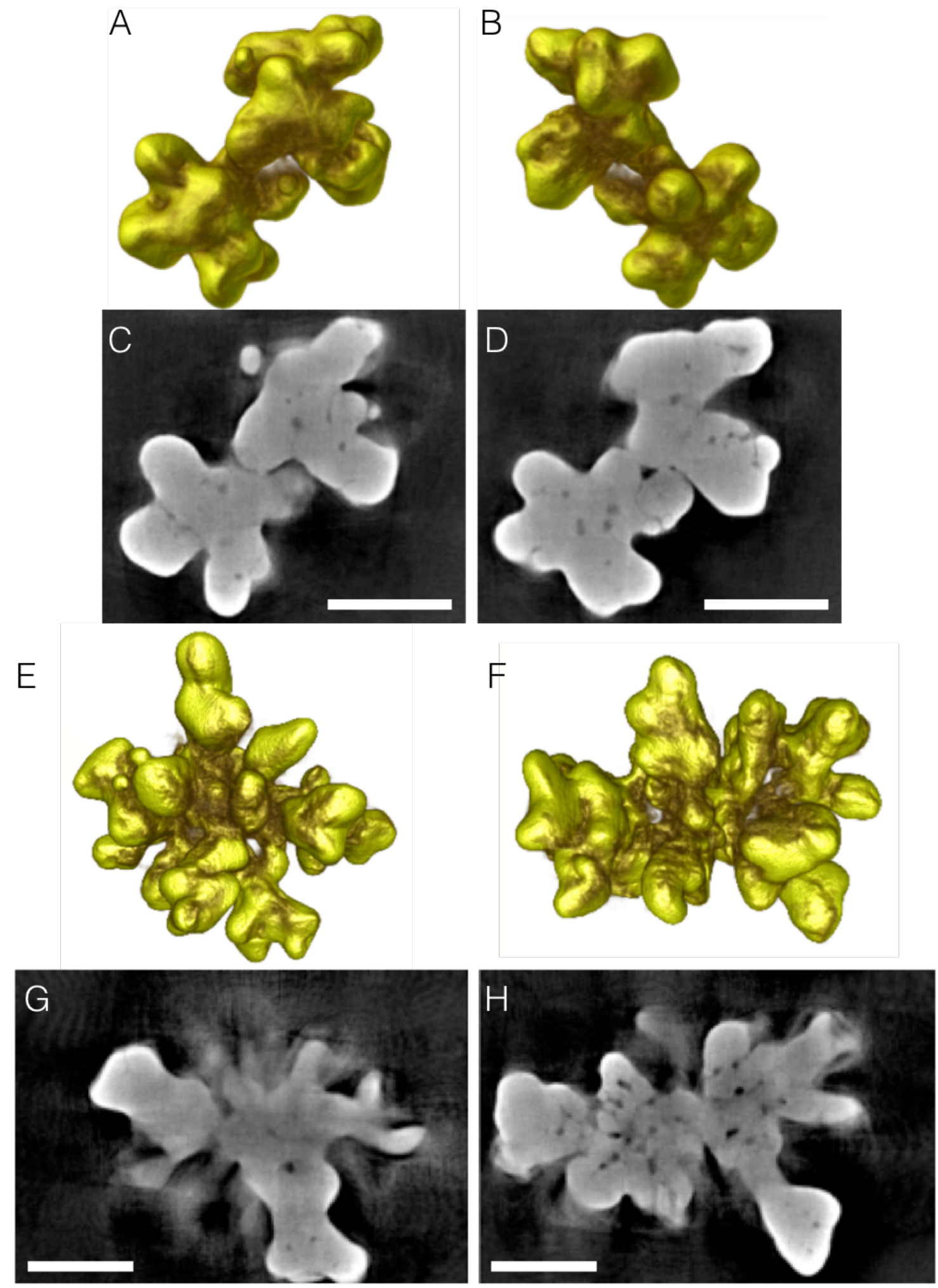

Figure 5. (A, B and $\boldsymbol{E}, \boldsymbol{F}) 3 D$ tomographic reconstruction along two different viewing directions of $(\boldsymbol{A}, \boldsymbol{B})$ two Au nanoparticle obtained at pH 4.1 and with $53.3 \mathrm{pM}$ Au seeds and (D, E) one AuNPs obtained at pH 4.1 and 
with 8.9 pM Au seeds $(\boldsymbol{E}, \boldsymbol{F})$. Different orthoslices through the reconstruction revealing the location of holes and defects in the Au nanostructures of NPs obtained at pH 4.1 and with 53.3 pM Au seeds (C, D) and 8.9 pM Au seeds (G, H) (Scale bar correspond with $50 \mathrm{~nm}$ in both cases)

The above results show a remarkable influence of the $\mathrm{pH}$ on the final morphology of the particles. Thus, working in a $\mathrm{pH}$ range of $2.5-5$ the particles show a well-defined morphology ranging from spherical to highly branched NPs. To summarize, to analyze the effect of $\mathrm{pH}$ in the particles growth, we need to take into consideration the following:

i) The $\mathrm{pH}$ influences the ionization degree of the PAH being its reported $\mathrm{pKa}$ values in the $\mathrm{pH}$ range $8-9 .{ }^{30}$ In fact, as weak cationic polyelectrolyte bearing primary amines, $\mathrm{PAH}$ can show from extended coil to segmental collapse conformational changes upon tuning its charge with the $\mathrm{pH}^{37}$ On the other hand, the $\mathrm{pH}$ and the presence of $\mathrm{Cl}^{-}$determine the speciation of gold(III) species in the solution. ${ }^{29-31}$ Thus, while at $\mathrm{pH}$ range 2.5 just $\mathrm{AuCl}_{4}^{-}$is present in the reaction medium, between 3 and 6 two species, $\mathrm{AuCl}_{4}^{-}$and $\mathrm{AuCl}_{3}(\mathrm{OH})^{-}$, coexist. ${ }^{31}$ Besides, although the most abundant species is the $\mathrm{AuCl}_{4}^{-}$, the amount $\mathrm{AuCl}_{3}(\mathrm{OH})^{-}$ gradually increases with the $\mathrm{pH}$.

ii) At the studied $\mathrm{pH}$ range $(2.5-5)$, the $\mathrm{PAH}$ is highly charged, therefore we expect the formation of ion pairs between the protonated amine group of PAH and the gold salt precursor $\left(\mathrm{AuCl}_{4}^{-} \text {or } \mathrm{AuCl}_{3}(\mathrm{OH})^{-}\right)^{34}$ which can also lead to the formation of colloidal aggregates. ${ }^{38}$ Interestingly, the formation of these ion pairs shifts their redox potential to more negative values, similar to what has been reported for quaternary ammonium salts. ${ }^{35}$

Based on the previous consideration, $\mathrm{PAH}-\mathrm{Au}(\mathrm{III})$ mixtures at different $\mathrm{pHs}$ (namely 2.5, 4.1 and 4.8) were analyzed by dynamic light scattering (DLS). Interestingly, the analysis indicates the presence of colloidal aggregates independently of the $\mathrm{pH}$ with average sizes 
ranging from $26 \mathrm{~nm}$ to $143 \mathrm{~nm}$ from $\mathrm{pH} 2.5$ to $\mathrm{pH} 4.8$, respectively (see Figure $\mathbf{S 1 5}$ and Table S1 in the SI). Therefore, we propose that the PAH-Au(III) mixtures, corresponding to a PAH monomer: $\mathrm{AuCl}_{4}{ }^{-}$ratio of 3.6, led to a decrease of the electrostatic repulsions between adjacent protonated amine units due to ion-pair formation and being responsible for the colloidal polymer aggregate formation.

iii) The formation of the ion-pair is corroborated by the absence of nucleation after adding the AA due to shifting the redox potential of the gold precursor to more negative values. The chemical reduction of the Au species is just triggered by the addition of gold seeds. Besides, the average size of the obtained particles can be modulated by the amount of seed solution added. This is in full agreement with previous reports on the seeded growth of Au NPs. ${ }^{10,16,35}$ At a fixed $\mathrm{pH}$, the size control is also accompanied by a tunability of the optical properties of the NPs from the visible to the NIR region (Figure 2), which is associated with their morphology, and particularly with the lengths and number of branches.

iv) The influence of the $\mathrm{pH}$ in the final morphology of the particles could be also ascribed in part to the $\mathrm{pH}$-dependent $\mathrm{AuCl}_{4}^{-}: \mathrm{AuCl}_{3}(\mathrm{OH})^{-}$ratio present in the reaction medium. Moreover, the kinetics of the process could be considered as fast with the gold salt reduction taking place in the first 3 minutes. Interestingly, once the gold salt is reduced a reshaping process occurs where the branched morphology is smoothed resulting in the narrowing and intensification of the LSRP band. Thus, the formation of size-dependent PAH$\mathrm{AuCl}_{4}{ }^{-}$aggregates as demonstrated by DLS may be responsible for the branched morphology of overgrown particles and the appearance of internal voids in the particles for highly branched nanoparticles (see Figure 4).

Next, we evaluated the sensing capabilities of this new type of branched AuNPs studying the shift in the LSPR band induced by a change in the local refractive index around 
metal nanostructures. ${ }^{39}$ Thus, the refractive index sensitivity (RIS), defined as the shift in the LSPR band with the variation of the refractive index of the medium $(\Delta \lambda / \Delta \mathrm{n})$, were measured by varying the refractive index of the solution using different water-glycerol mixtures (see Figure 6A and further details in Figures S16 and S17 in SI). Three different types of branched NPs with LSPR bands located at $538 \mathrm{~nm}, 600 \mathrm{~nm}$ and $709 \mathrm{~nm}$ were studied (see Figure S16). All the samples show a reasonably good linear dependence of the LSPR shift with the refractive index (see Figure S17). The RIS determined from the linear fit were 95, 163 and $305 \mathrm{~nm} / \mathrm{RIU}$ for the AuNPs with the LSPR centered at ca. $538 \mathrm{~nm}, 600 \mathrm{~nm}$ and $709 \mathrm{~nm}$, respectively. As expected, the branched AuNPs with longer LSPR presented higher sensitivity and with values similar consistent with studies on other anisotropic NPs. ${ }^{16,40}$ Interestingly, the reliability of LSPR sensing, and therefore the RIS, can be improved by analyzing the curvature of the LSPR band. The curvature of the LSPR band is characterized by the peak maximum and two inflections points. The two inflection points can be easily obtained since they appear as local maxima/minima in the first-order derivative and have a 0 value in the second-order derivative spectrum (see red circles in Figure 6B), with the inflection point at the red side of the LSPR band showing a higher RIS than that obtained by the peak maximum. ${ }^{41}$ Figure $\mathbf{6 C}$ shows the RIS measured for the three types of branched $\mathrm{Au}$ NPs at the peak maximum and the inflection point at longer wavelengths. In the three cases, the inflection point presents a better RIS performance, with an improvement ranging from $11.5 \%$ to $24.5 \%$ which is similar to that reported for other Au nanoparticles. ${ }^{41}$ 

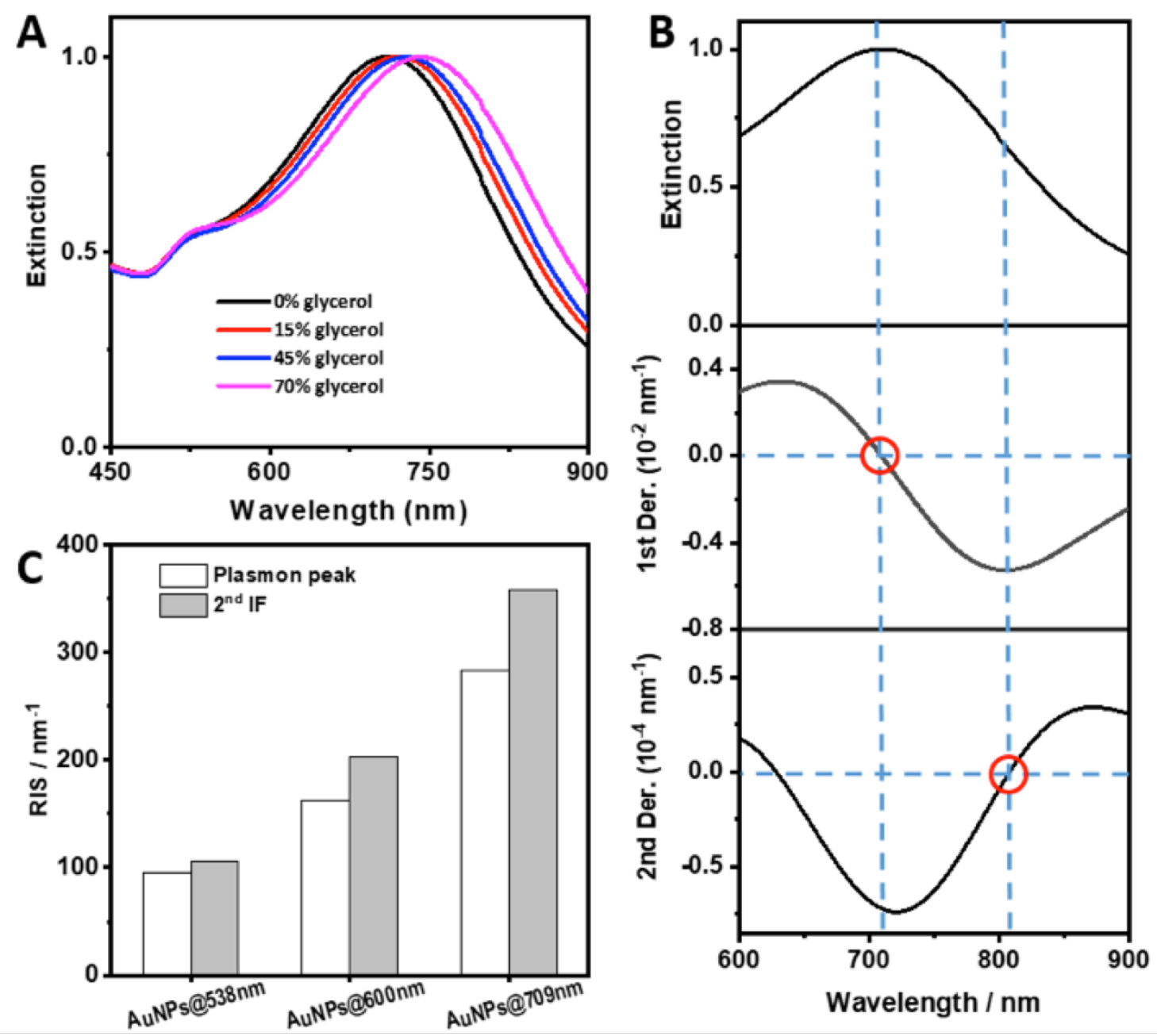

Figure 6. (A) Extinction spectra of branched AuNPs dispersed in different water-glycerol mixtures as indicated with the LSPR centered at $709 \mathrm{~nm}$ in water. (B) Representative extinction (top), first derivative (middle), and second derivative (bottom )spectra of branched AuNPs. The dashed blue lines are a guide for the eye. The red circles indicate the LSPR band maximum, indicated by the 0 value of the first-order derivative spectrum, and the inflection point at longer wavelengths of the extinction spectrum, which shows a 0 value in the second-order derivative spectrum. (C) Refractive index sensitivities were obtained from the LSPR peak maximum and the inflection point at longer wavelengths for the three types of branched NPs studied.

Finally, one of the key issues for the applicability of the Au nanoparticles is the ease of surface functionalization. To demonstrate that, we carried out as a proof of concept the functionalization of the as-synthesized AuNPs with polyacrylic acid-co-maleic acid 
(PAcMA) via NHS/EDC chemistry. Thus the carboxylic acid functional groups from PAcMA will form an amide bond with the amine groups from PAH. ${ }^{42,43}$ After the functionalization the characteristic LSRP band of Au branched NPs exhibited a slight redshift $(c a .10 \mathrm{~nm})$ that can be explained by changes in the dielectric medium surrounding the NPs after functionalization (see Figure S18 in the SI). ${ }^{44}$ Additionally, the $\zeta$-potential of the Au NPs, initially highly positive, became highly negative $(-48.4 \mathrm{~mW})$ due to the carboxylic moieties of the PAcMA polymer. Moreover, the presence of PAcMA on the NPs surface was also demonstrated by FTIR spectroscopy (see Figure S19 in the SI). Interestingly, the PAcMA functionalization confer a high stability to the particles even in phosphate buffer saline $(\mathrm{PBS}, \mathrm{pH}=7.6,[\mathrm{Phosphate}]=100 \mathrm{mM},[\mathrm{NaCl}]=200 \mathrm{mM})$. The surface modification with PAcMA through electrostatic interactions led to limited stability in PBS as demonstrated by the overall increase in the intensity of the extinction spectrum together with the broadening of the LSPR band (see Figure S20 in the SI).

\section{Conclusions}

We have developed a seed-mediated synthesis of branched AuNPs in the presence of PAH that offers tight control over the optical properties in the Vis-NIR $(560-1260 \mathrm{~nm})$ region. Relevant aspects of this synthesis are the possibility of taking advantage of the PAH-Au(III) interaction at different $\mathrm{pH}$ to drive the growth of NPs over small pre-synthesized gold seeds to control the size and branching morphology of AuNPs formed, thus covering a wide wavelength range in visible and near-infrared. Advanced electron microscopy techniques demonstrated the polycrystalline nature of the NPs and facilitated a better understanding of branched morphology. The mechanism of nanostructure formation was explored. A Kinetically controlled growth at early stages, probably due to preferential growth of the twin 
boundaries of spherical seeds with a multi-twinned crystallographic structure is proposed. Additionally, the LSPR sensing capabilities of this new type of particles were evaluated by measuring the refractive index sensitivity by the inflection point. Furthermore, the versatility of the PAH chemistry allowed the easy functionalization of the synthesized NPs. We hope that the present work will drive the development of new anisotropic nanoparticles tailored for particular applications and suggest new future directions to explore in branched AuNPs synthesis using polyamines.

\section{Experimental section}

\section{Materials}

Gold(III) chloride trihydrate $\left(\mathrm{HAuCl}_{4} \cdot 3 \mathrm{H}_{2} \mathrm{O}, 99.9 \%\right)$, trisodium citrate dihydrate $\left(\mathrm{C}_{6} \mathrm{H}_{5} \mathrm{Na}_{3} \mathrm{O}_{7} \cdot 2 \mathrm{H}_{2} \mathrm{O}, \geq 99.5 \%\right)$, sodium borohydride $\left(\mathrm{NaBH}_{4}\right.$, ReagentPlus $\left.{ }^{\circledR} 99 \%\right)$, L-ascorbic acid $\left(\mathrm{C}_{6} \mathrm{H}_{8} \mathrm{O}_{6}\right.$, BioXtra $\geq 99.0 \%$ crystalline $)$, poly(allylamine hydrochloride) $\left(\left[\mathrm{CH}_{2} \mathrm{CH}\left(\mathrm{CH}_{2} \mathrm{NH}_{2} \cdot \mathrm{HCl}\right)\right]_{\mathrm{n}}\right.$, average $\mathrm{Mw} 50,000$ (GPC vs. PEG std.)), sodium hydroxide $(\mathrm{NaOH}$, BioXtra $\geq 98 \%$, pellets, anhydrous) hydrochloric acid ( $\mathrm{HCl}$, ACS reagent, 37\%), N-(3dimethylaminopropyl)- $N^{\prime}$-ethylcarbodiimide hydrochloride (EDC), poly(acrylic acid-co-maleic acid) solution (PAcMA, average Mw 3,000, 50 wt. \% in $\mathrm{H}_{2} \mathrm{O}$ ) were obtained from Sigma-Aldrich. N-Hydroxysuccinimide (NHS, 98+\%) was obtained from Alfa Aesar. All reagents were used as received without further purification. Ultrapure water (type I) was used for the preparation of all the water-based solutions. The glassware was cleaned with aqua regia prior to the experiments.

\section{Methods}

Synthesis of gold seeds. The synthesis of the gold seeds was based on a previous report with a few modifications. ${ }^{34}$ Briefly, $20 \mathrm{~mL}$ of an aqueous solution containing $0.125 \mathrm{mM} \mathrm{HAuCl}_{4}$ and $0.25 \mathrm{mM}$ trisodium citrate was prepared in a round-bottomed flask at RT. Under vigorous stirring, $300 \mu \mathrm{L}$ of an ice-cold freshly 
prepared $0.01 \mathrm{M} \mathrm{NaBH}_{4}$ solution was rapidly injected. Vigorous stirring was maintained for 15 more seconds and then slowed down for $15 \mathrm{~min}$ at RT. The seed solution was heated 60 minutes at $40{ }^{\circ} \mathrm{C}$ with slow magnetic stirring and then cooled with continued stirring to ensure the removal of excess $\mathrm{NaBH}_{4}$ before use. The seed solution was diluted to a final concentration of $0.1 \mathrm{mM}$ in Au metal for the NP growth experiments.

Preparation of poly(allylamine hydrochloride) solution. $1 \mathrm{mg} \mathrm{mL}^{-1} \mathrm{PAH}$ solution was prepared in water (5 mM $\mathrm{HCl}$ ). After vigorous stirring, the PAH solution was left undisturbed for a minimum of 3 days for complete solubilization of the polymer. ${ }^{45}$

Synthesis of branched AuNPs. In a round bottom reaction flask containing a certain volume of Milli-Q water (ca. $17 \mathrm{~mL}), 2 \mathrm{~mL}$ of PAH $\left(1 \mathrm{mg} \cdot \mathrm{mL}^{-1}\right)$ and $0.3 \mathrm{~mL}$ of $\mathrm{HAuCl}_{4}(20 \mathrm{mM})$ were added under vigorous magnetic stirring. After that, the $\mathrm{pH}$ was adjusted with either $\mathrm{NaOH}$ or $\mathrm{HCl}(0.2 \mathrm{M})$ solution. Thus, to adjust the $\mathrm{pH}$ to 2.5, $0.2 \mathrm{~mL}$ of $\mathrm{HCl}(0.2 \mathrm{M})$ was added to the aqueous solution. Instead, for $\mathrm{pHs}$ between 3.5 and 7.3, volumes between 0.1 and $0.3 \mathrm{~mL}$ of $\mathrm{NaOH}(0.2 \mathrm{M})$ were added. After 5 minutes of vigorous stirring, $0.5 \mathrm{~mL}$ of AA (20 $\mathrm{mM})$ was added and subsequently a certain amount of gold seeds $\left(\left[\mathrm{Au}^{0}\right]=0.1 \mathrm{mM}\right)$. The total volume was 20 $\mathrm{mL}$ regardless of the amount of seed added. Experiments with final NP concentrations of Au seeds of $8.9 \mathrm{pM}$, $13.3 \mathrm{pM}, 17.8 \mathrm{pM}, 26.7 \mathrm{pM}, 35.4 \mathrm{pM}, 53.3 \mathrm{pM}, 71.1 \mathrm{pM}, 106.6 \mathrm{pM}$ were carried out. The reactions were kept for $4 \mathrm{~h}$ at $22^{\circ} \mathrm{C}$ under vigorous stirring. Then the NPs were purified by centrifugation (between 1500 and 3500 rpm, depending on the size) re-suspended in miliQ water.

Arresting the reaction. To monitor the progress of the reaction carried out at $\mathrm{pH} 4.1$ and $53.3 \mathrm{pM}$ of seeds, 3 $\mathrm{mL}$ aliquots were extracted from the reaction at various times and added to $2 \mathrm{~mL}$ of aqueous PEG-SH solution $\left(1 \mathrm{mg} \mathrm{mL}^{-1}\right)$ and then vigorously stirred. After $30 \mathrm{~min}$. the NPs were isolated by centrifugation at $9000 \mathrm{rpm}$, then resuspended in $1.5 \mathrm{~mL}$ of Milli-Q water for transmission electron microscopy (TEM) and Vis-NIR spectroscopy analysis.

Functionalization of AuNPs with poly(acrylic acid-co-maleic acid). PAcMA was activated with EDC/NHS before adding it to the AuNPs. Briefly, to $5 \mathrm{~mL}$ of an aqueous solution containing $6 \mu \mathrm{L}$ ( 50 wt. $\%$ in $\mathrm{H}_{2} \mathrm{O}$ ) of PAcMA, $5 \mathrm{~mL}$ of $30 \mathrm{mM}$ EDC and $5 \mathrm{~mL}$ of $30 \mathrm{mM}$ NHS were added. The reaction was maintained under ultrasound for 15 minutes then vigorous magnetic stirring for $2 \mathrm{~h}$. The activated polymer solution was dripped 
onto the NP reaction, which was vigorously stirred for $4 \mathrm{~h}$. (Note: During the course of reaction, the NPs coalesced even under magnetic stirring, so the NPs were re-suspended using ultrasound to ensure a homogeneous solution) Finally, the AuNPs were washed repeatedly with PBS $(\mathrm{pH}=7.4)$ and water.

\section{Characterization}

The extinction spectra were recorded using a JASCO 770 UV-Vis-NIR spectrophotometer provided by the PROTEOMASS-BIOSCOPE facility (Caparica, Portugal). All spectra were recorded using a HELMA $1 \mathrm{~cm}$ light path quartz cell. Dynamic light scattering (DLS) and $\zeta$-potential analysis were carried out in a Malvern ZS instrument at $22{ }^{\circ} \mathrm{C}$ in standard $1 \mathrm{~mL}$ polystyrene cuvettes provided by the PROTEOMASS-BIOSCOPE facility (Caparica, Portugal). DLS measurements were obtained with $180 \mathrm{sec}$ of equilibration time. Fourier transform infrared (FT-IR) analysis was performed using a Bruker Tensor 27 instrument in $\mathrm{KBr}$ or $\mathrm{NaCl}$ discs and with a resolution of $4 \mathrm{~cm}^{-1}$. Low magnification transmission electron microscopy (TEM) images were obtained using a JEOL JEM 1010 TEM microscope, working at $100 \mathrm{kV}$. A JEOL JEM 2010F field-emission gun TEM working at $200 \mathrm{kV}$ was used to obtain HRTEM images. Particle size and mean size distribution were calculated from TEM micrographs using the ImageJ package. High angle annular dark-field scanning transmission electron microscopy (HAADF-STEM) images and electron tomography series were acquired using a Probe-Corrected FEI Titan G2 $80-200 \mathrm{kV}$ ChemiSTEM. A dual-axis tomography with $\pm 70^{\circ}$ was performed in both axes to minimize the missing wedge. Gold fiducials of $10 \mathrm{~nm}$ in size were deposited on the grids before depositing the AuNPs to allow for highly accurate tracking and reconstruction, and the acquisition was carried out with an 8 mrad convergence angle.

\section{Supporting Information}

Movie S1. Ortho slices through the reconstruction of the particles made with $53.3 \mathrm{pM}$ of gold seed solution. (AVI).

Movie S2. Ortho slices through the reconstruction of the particles made with $8.9 \mathrm{pM}$ of gold seed solution. (AVI)

Movie S3. Rotation of the 3D visualization of the particles made with $53.3 \mathrm{pM}$ of gold seed solution. (AVI)

Movie S4. Rotation of the 3D visualization of the particles made with $8.9 \mathrm{pM}$ of gold seed solution. (AVI) 
NPs Characterization details; HRTEM images of AuNPs, HRTEM images of Au seeds; zeta-potential measurements; Time evolution of extinction spectra of AuNP, TEM images of arrested intermediates; 3D STEM tomographic reconstruction of two different nanoparticles along two different viewing directions of the Au nanoparticles; Dynamic light scattering (DLS) analysis of PAH-Au(III) at different pHs; Extinction spectra of branched AuNPs dispersed in different water-glycerol mixtures; Inflection point method for three branched AuNPs dispersed in different water-glycerol mixtures; FTIR Spectra of PAcMA vs AuNPs@PAH@PAcMA (PDF).

\section{Author Information}

\section{Corresponding Author}

Javier Fernández-Lodeiro - BIOSCOPE Group, LAQV@REQUIMTE, Chemistry Department, NOVA School of Science and Technology, NOVA University Lisbon, Caparica Campus, 2829-516 Caparica, Portugal. PROTEOMASS Scientific Society, Rua dos Inventores, Madam Parque, Caparica Campus, 2829-516 Caparica, Portugal; orcid.org/0000-0001-8853-2631; Email: j.lodeiro@,fct.unl.pt

Carlos Lodeiro - BIOSCOPE Group, LAQV@REQUIMTE, Chemistry Department, NOVA School of Science and Technology, NOVA University Lisbon, Caparica Campus, 2829-516 Caparica, Portugal. PROTEOMASS Scientific Society, Rua dos Inventores, Madam Parque, Caparica Campus, 2829-516 Caparica, Portugal; orcid.org/0000-0001-5582-5446; Email: cle@ffct.unl.pt | clodeiro@bioscopegroup.org

\section{Authors}


Silvia Nuti ₹-BIOSCOPE Group, LAQV@REQUIMTE, Chemistry Department, NOVA

School of Science and Technology, NOVA University Lisbon, Caparica Campus, 2829-516

Caparica, Portugal.; orcid.org/0000-0003-1420-6109

Carlos Fernández-Lodeiro $\$$ - CINBIO, Universidade de Vigo, Departamento de Química Física, Campus Universitario Lagoas Marcosende, 36310 Vigo, Spain. Galicia Sur Health Research Institute (IIS Galicia Sur), SERGAS-UVIGO, 36310 Vigo, Spain; orcid.org/0000-0002-8331-8799.

Adrián Fernández-Lodeiro - BIOSCOPE Group, LAQV@REQUIMTE, Chemistry Department, NOVA School of Science and Technology, NOVA University Lisbon, Caparica Campus, 2829-516 Caparica, Portugal. PROTEOMASS Scientific Society, Rua dos Inventores, Madam Parque, Caparica Campus, 2829-516 Caparica, Portugal; orcid.org/0000-0003-3742-1186.

Jorge Pérez-Juste - CINBIO, Universidade de Vigo, Departamento de Química Física, Campus Universitario Lagoas Marcosende, 36310 Vigo, Spain. Galicia Sur Health Research Institute (IIS Galicia Sur), SERGAS-UVIGO, 36310 Vigo, Spain; orcid.org/0000-0002-4614-1699.

Isabel Pastoriza-Santos - CINBIO, Universidade de Vigo, Departamento de Química Física, Campus Universitario Lagoas Marcosende, 36310 Vigo, Spain. Galicia Sur Health Research Institute (IIS Galicia Sur), SERGAS-UVIGO, 36310 Vigo, Spain; orcid.org/0000-0002-1091-1364.

Alec P. LaGrow - International Iberian Nanotechnology Laboratory, Braga, 4715-330, Portugal; orcid.org/0000-0002-3306-6458.

Olivier Schraidt - International Iberian Nanotechnology Laboratory, Braga, 4715-330, Portugal; orcid.org/0000-0001-8114-3713.

Jose Luis Capelo-Martínez - BIOSCOPE Group, LAQV@REQUIMTE, Chemistry Department, NOVA School of Science and Technology, NOVA University Lisbon, Caparica Campus, 2829-516 Caparica, Portugal. PROTEOMASS Scientific Society, Rua dos Inventores, Madam Parque, Caparica Campus, 2829-516 Caparica, Portugal; orcid.org/0000-0001-6276-8507. 


\section{Author Contributions}

S.N., C.F-L, J.F-L and A.F-L performed the experiments, data analysis, the calculations, and wrote the first manuscript draft. C. F-L and J. F-L perform Low-Resolution TEM analysis. J. P-J, I. P-S and J. L. C-M, C. L, helped with useful tips regarding the experimental data analysis. A. P. L-G and O. S. provided the HRTEM, HAADF STEM and Tomography analysis. J. F-L, C.L, J. P. J, I. P-S and J. L C-M. obtained the funding for the project. All authors contributed to writing and correction of final draft, given approval for the final version of the manuscript.

†These authors contributed equally.

\section{Funding Sources}

The authors acknowledge the financial support by the Associate Laboratory Research Unit for Green Chemistry-Clean Processes and Technologies-LAQV which is financed by national funds from FCT/MEC (UID/QUI/50006/2013) and co-financed by the ERDF under the PT2020 Partnership Agreement (POCI-010145-FEDER-007265), as well as the PROTEOMASS Scientific Society General Funds (Portugal) for funding support. S.N.; J.F.-L., A.F.-L.; C.L. and J.L.C.-M. thank the FCT-MEC for the research grant SiSi4Bacter (PTDC/QUI-COL/1517/2020). I. P.-S. and J.P.-J. acknowledge financial support from Spanish MINECO grant PID2019-108954RB-I00.

\section{Acknowledgments}

J.F.-L. thanks the FCT-UNL for the DL57/2016 Assistant Researcher Contract. S.N. thanks the FCT-MEC Portugal for her doctoral grant associated with the Chemistry PhD program (SFRH/BD/144618/2019). A.F.L. thanks the FCT-UNL for the DL57/2021 Assistant Researcher Contract under the grant PTDC/QUICOL/1517/2020, and C. F.-L. acknowledges Xunta de Galicia for a predoctoral scholarship (Programa de axudas á etapa predoutoral). 


\section{References}

(1) Ortiz-Castillo, J. E.; Gallo-Villanueva, R. C.; Madou, M. J.; Perez-Gonzalez, V. H. Anisotropic Gold Nanoparticles: A Survey of Recent Synthetic Methodologies. Coord. Chem. Rev. 2020, 425, 213489.

(2) Boken, J.; Khurana, P.; Thatai, S.; Kumar, D.; Prasad, S. Plasmonic Nanoparticles and Their Analytical Applications: A Review. Appl. Spectrosc. Rev. 2017, 52 (9), 774-820.

(3) Reguera, J.; Langer, J.; Jiménez de Aberasturi, D.; Liz-Marzán, L. M. Anisotropic Metal Nanoparticles for Surface Enhanced Raman Scattering. Chem. Soc. Rev. 2017, 46 (13), 3866-3885..

(4) Kohout, C.; Santi, C.; Polito, L. Anisotropic Gold Nanoparticles in Biomedical Applications. Int. J. Mol. Sci. 2018, 19 (11), 3385.

(5) Venditti, I. Engineered Gold-Based Nanomaterials: Morphologies and Functionalities in Biomedical Applications. A Mini Review. Bioengineering 2019, 6 (2), 53.

(6) Priecel, P.; Adekunle Salami, H.; Padilla, R. H.; Zhong, Z.; Lopez-Sanchez, J. A. Anisotropic Gold Nanoparticles: Preparation and Applications in Catalysis. Chinese J. Catal. 2016, 37 (10), 16191650.

(7) Gonçalves, A. S. C.; Rodrigues, C. F.; Moreira, A. F.; Correia, I. J. Strategies to Improve the Photothermal Capacity of Gold-Based Nanomedicines. Acta Biomater. 2020, 116, 105-137.

(8) Rodríguez-Lorenzo, L.; Álvarez-Puebla, R. A.; Pastoriza-Santos, I.; Mazzucco, S.; Stéphan, O.; Kociak, M.; Liz-Marzán, L. M.; García de Abajo, F. J. Zeptomol Detection Through Controlled Ultrasensitive Surface-Enhanced Raman Scattering. J. Am. Chem. Soc. 2009, 131 (13), 4616-4618.

(9) Mousavi, S. M.; Zarei, M.; Hashemi, S. A.; Ramakrishna, S.; Chiang, W.-H.; Lai, C. W.; Gholami, A. Gold Nanostars-Diagnosis, Bioimaging and Biomedical Applications. Drug Metab. Rev. 2020, 52 (2), $299-318$.

(10) Chang, Y.-X.; Zhang, N.-N.; Xing, Y.-C.; Zhang, Q.; Oh, A.; Gao, H.-M.; Zhu, Y.; Baik, H.; Kim, B.; Yang, Y.; Chang, W.-S.; Sun, T.; Zhang, J.; Lu, Z.-Y.; Lee, K.; Link, S.; Liu, K. Gold Nanotetrapods with Unique Topological Structure and Ultranarrow Plasmonic Band as Multifunctional Therapeutic Agents. J. Phys. Chem. Lett. 2019, 10 (16), 4505-4510.

(11) Casu, A.; Cabrini, E.; Donà, A.; Falqui, A.; Diaz-Fernandez, Y.; Milanese, C.; Taglietti, A.; 
Pallavicini, P. Controlled Synthesis of Gold Nanostars by Using a Zwitterionic Surfactant. Chem. - A Eur. J. 2012, 18 (30), 9381-9390.

(12) Atta, S.; Beetz, M.; Fabris, L. Understanding the Role of AgNO 3 Concentration and Seed Morphology in the Achievement of Tunable Shape Control in Gold Nanostars. Nanoscale 2019, 11 (6), 2946-2958.

(13) Pallavicini, P.; Donà, A.; Casu, A.; Chirico, G.; Collini, M.; Dacarro, G.; Falqui, A.; Milanese, C.; Sironi, L.; Taglietti, A. Triton X-100 for Three-Plasmon Gold Nanostars with Two Photothermally Active NIR (near IR) and SWIR (Short-Wavelength IR) Channels. Chem. Commun. 2013, 49 (56), 6265.

(14) Senthil Kumar, P.; Pastoriza-Santos, I.; Rodríguez-González, B.; Javier García de Abajo, F.; LizMarzán, L. M. High-Yield Synthesis and Optical Response of Gold Nanostars. Nanotechnology 2008, $19(1), 015606$.

(15) Pazos-Perez, N.; Guerrini, L.; Alvarez-Puebla, R. A. Plasmon Tunability of Gold Nanostars at the Tip Apexes. ACS Omega 2018, 3 (12), 17173-17179.

(16) M. Pallares, R.; Stilson, T.; Choo, P.; Hu, J.; Odom, T. W. Using Good's Buffers To Control the Anisotropic Structure and Optical Properties of Spiky Gold Nanoparticles for Refractive Index Sensing. ACS Appl. Nano Mater. 2019, 2 (8), 5266-5271.

(17) Yuan, H.; Khoury, C. G.; Hwang, H.; Wilson, C. M.; Grant, G. A.; Vo-Dinh, T. Gold Nanostars: Surfactant-Free Synthesis, 3D Modelling, and Two-Photon Photoluminescence Imaging. Nanotechnology 2012, 23 (7), 075102.

(18) De Silva Indrasekara, A. S.; Johnson, S. F.; Odion, R. A.; Vo-Dinh, T. Manipulation of the Geometry and Modulation of the Optical Response of Surfactant-Free Gold Nanostars: A Systematic Bottom-Up Synthesis. ACS Omega 2018, 3 (2), 2202-2210.

(19) Van de Broek, B.; Frederix, F.; Bonroy, K.; Jans, H.; Jans, K.; Borghs, G.; Maes, G. ShapeControlled Synthesis of NIR Absorbing Branched Gold Nanoparticles and Morphology Stabilization with Alkanethiols. Nanotechnology 2011, 22 (1), 015601.

(20) Fabris, L. Gold Nanostars in Biology and Medicine: Understanding Physicochemical Properties to Broaden Applicability. J. Phys. Chem. C 2020, 124 (49), 26540-26553. 
(21) Wang, Y.; Asefa, T. Poly(Allylamine)-Stabilized Colloidal Copper Nanoparticles: Synthesis, Morphology, and Their Surface-Enhanced Raman Scattering Properties. Langmuir 2010, 26 (10), $7469-7474$.

(22) Fu, G.; Jiang, X.; Gong, M.; Chen, Y.; Tang, Y.; Lin, J.; Lu, T. Highly Branched Platinum Nanolance Assemblies by Polyallylamine Functionalization as Superior Active, Stable, and Alcohol-Tolerant Oxygen Reduction Electrocatalysts. Nanoscale 2014, 6 (14), 8226-8234.

(23) Sardar, R.; Bjorge, N. S.; Shumaker-Parry, J. S. PH-Controlled Assemblies of Polymeric AmineStabilized Gold Nanoparticles. Macromolecules 2008, 41 (12), 4347-4352.

(24) Sardar, R.; Park, J.-W.; Shumaker-Parry, J. S. Polymer-Induced Synthesis of Stable Gold and Silver Nanoparticles and Subsequent Ligand Exchange in Water. Langmuir 2007, 23 (23), 11883-11889.

(25) Liu, Z.; Fu, G.; Tang, Y.; Sun, D.; Chen, Y.; Lu, T. A Facile, One-Pot Synthesis of Highly Branched Au Nanocorals and Their Enhanced Electrocatalytic Activity for Ethanol Oxidation. CrystEngComm 2014, $16(36), 8576-8581$.

(26) Pereira, S. O.; Barros-Timmons, A.; Trindade, T. Biofunctionalisation of Colloidal Gold Nanoparticles via Polyelectrolytes Assemblies. Colloid Polym. Sci. 2014, 292 (1), 33-50.

(27) Masereel, B.; Dinguizli, M.; Bouzin, C.; Moniotte, N.; Feron, O.; Gallez, B.; Vander Borght, T.; Michiels, C.; Lucas, S. Antibody Immobilization on Gold Nanoparticles Coated Layer-by-Layer with Polyelectrolytes. J. Nanoparticle Res. 2011, 13 (4), 1573-1580.

(28) Martins, G.; Fernández-Lodeiro, J.; Djafari, J.; Lodeiro, C.; Capelo, J. L.; Santos, H. M. Label-Free Protein Quantification after Ultrafast Digestion of Complex Proteomes Using Ultrasonic Energy and Immobilized-Trypsin Magnetic Nanoparticles. Talanta 2019, 196 (December 2018), 262-270.

(29) Tu, Y.-J.; Njus, D.; Schlegel, H. B. A Theoretical Study of Ascorbic Acid Oxidation and HOO \% O 2 - Radical Scavenging. Org. Biomol. Chem. 2017, 15 (20), 4417-4431.

(30) Choi, J.; Rubner, M. F. Influence of the Degree of Ionization on Weak Polyelectrolyte Multilayer Assembly. Macromolecules 2005, 38 (1), 116-124.

(31) Hönes, G., Huisl, W., Keim, R., Schwager, B. Gmelin Handbook of Inorganic and Organometallic Chemistry - 8th Edition; Edition, 8th, Ed.; Springer-Verlag, 1996; Vol. 15.

(32) Kumar, A.; Mandal, S.; Selvakannan, P. R.; Pasricha, R.; Mandale, A. B.; Sastry, M. Investigation 
into the Interaction between Surface-Bound Alkylamines and Gold Nanoparticles. Langmuir 2003, 19 (15), 6277-6282.

(33) Murphy, C. J.; Thompson, L. B.; Chernak, D. J.; Yang, J. A.; Sivapalan, S. T.; Boulos, S. P.; Huang, J.; Alkilany, A. M.; Sisco, P. N. Gold Nanorod Crystal Growth: From Seed-Mediated Synthesis to Nanoscale Sculpting. Curr. Opin. Colloid Interface Sci. 2011, 16 (2), 128-134.

(34) Pérez-Juste, J.; Liz-Marzán, L. M.; Carnie, S.; Chan, D. Y. C.; Mulvaney, P. Electric-Field-Directed Growth of Gold Nanorods in Aqueous Surfactant Solutions. Adv. Funct. Mater. 2004, 14 (6), 571579.

(35) Rodríguez-Fernández, J.; Pérez-Juste, J.; García De Abajo, F. J.; Liz-Marzán, L. M. Seeded Growth of Submicron Au Colloids with Quadrupole Plasmon Resonance Modes. Langmuir 2006, 22 (16), $7007-7010$.

(36) Scarabelli, L.; Sánchez-Iglesias, A.; Pérez-Juste, J.; Liz-Marzán, L. M. A “Tips and Tricks” Practical Guide to the Synthesis of Gold Nanorods. J. Phys. Chem. Lett. 2015, 6 (21), 4270-4279.

(37) Kirwan, L. J.; Papastavrou, G.; Borkovec, M.; Behrens, S. H. Imaging the Coil-to-Globule Conformational Transition of a Weak Polyelectrolyte by Tuning the Polyelectrolyte Charge Density. Nano Lett. 2004, 4 (1), 149-152.

(38) Murthy, V. S.; Rana, R. K.; Wong, M. S. Nanoparticle-Assembled Capsule Synthesis: Formation of Colloidal Polyamine-Salt Intermediates. J. Phys. Chem. B 2006, 110 (51), 25619-25627.

(39) Sepúlveda, B.; Angelomé, P. C.; Lechuga, L. M.; Liz-Marzán, L. M. LSPR-Based Nanobiosensors. Nano Today 2009, 4 (3), 244-251.

(40) Barbosa, S.; Agrawal, A.; Rodríguez-Lorenzo, L.; Pastoriza-Santos, I.; Alvarez-Puebla, R. A.; Kornowski, A.; Weller, H.; Liz-Marzán, L. M. Tuning Size and Sensing Properties in Colloidal Gold Nanostars. Langmuir 2010, 26 (18), 14943-14950.

(41) Chen, P.; Tran, N. T.; Wen, X.; Xiong, Q.; Liedberg, B. Inflection Point of the Localized Surface Plasmon Resonance Peak: A General Method to Improve the Sensitivity. ACS Sensors 2017, 2 (2), $235-242$.

(42) Nakajima, N.; Ikada, Y. Mechanism of Amide Formation by Carbodiimide for Bioconjugation in Aqueous Media. Bioconjug. Chem. 1995, 6 (1), 123-130. 
(43) Tsai, T.-C.; Liu, C.-W.; Wu, Y.-C.; Ondevilla, N. A. P.; Osawa, M.; Chang, H.-C. In Situ Study of EDC/NHS Immobilization on Gold Surface Based on Attenuated Total Reflection Surface-Enhanced Infrared Absorption Spectroscopy (ATR-SEIRAS). Colloids Surfaces B Biointerfaces 2019, 175 (December 2018), 300-305.

(44) Schaming, D.; Nguyen, V.-Q.; Martin, P.; Lacroix, J.-C. Tunable Plasmon Resonance of Gold Nanoparticles Functionalized by Electroactive Bisthienylbenzene Oligomers or Polythiophene. $J$. Phys. Chem. C 2014, 118 (43), 25158-25166.

(45) Park, J.; Choi, Y. W.; Kim, K. B.; Chung, H.; Sohn, D. Aggregation Processes of a Weak Polyelectrolyte, Poly(Allylamine) Hydrochloride. Bull. Korean Chem. Soc. 2008, 29 (1), 104-110. 


\section{Polyallylamine assisted synthesis of 3D branched AuNPs with plasmon tunability in the vis-NIR region modification}

Silvia Nuti, ${ }^{\mathrm{a}, \mathrm{b}, \#}$ Carlos Fernández-Lodeiro, ${ }^{\mathrm{c}, \mathrm{d}, \#}$ Javier Fernández-Lodeiro, ${ }^{* \mathrm{a}, \mathrm{b}}$ Adrián Fernández-

Lodeiro, ${ }^{\mathrm{a}, \mathrm{b}}$ Jorge Pérez-Juste, ${ }^{\mathrm{c}, \mathrm{d}}$ Isabel Pastoriza-Santos, ${ }^{\mathrm{c.d}}$ Alex P. LaGrow, ${ }^{\mathrm{e}}$ Olivier Schraidt, ${ }^{\mathrm{e}}$ José

Luis Capelo-Martínez, ${ }^{\text {a,b }}$ Carlos Lodeiro.*a,b

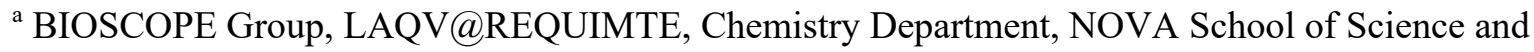
Technology, NOVA University Lisbon, Caparica Campus, 2829-516 Caparica, Portugal

${ }^{b}$ PROTEOMASS Scientific Society, Rua dos Inventores, Madam Parque, Caparica Campus, 2829516 Caparica, Portugal

${ }^{\mathrm{c}}$ CINBIO, Universidade de Vigo, Departamento de Química Física, Campus Universitario Lagoas Marcosende, 36310 Vigo, Spain

${ }^{\mathrm{d}}$ Galicia Sur Health Research Institute (IIS Galicia Sur), SERGAS-UVIGO, 36310 Vigo, Spain

${ }^{\mathrm{e}}$ International Iberian Nanotechnology Laboratory, Braga, 4715-330, Portugal

${ }^{\#}$ These authors contributed equally to this work 


\section{Supporting Information}

\section{Content}

\section{1.- Figures}

Figure S1. (A-C) HRTEM images of Au seeds after aging for $1 \mathrm{~h}$ at $40{ }^{\circ} \mathrm{C}$. (D) Vis-NIR spectrum of AuNPs seeds in water.

Figure S2. Vis-NIR spectra of AuNPs obtained adding $53.3 \mathrm{pM}$ seeds (in terms of NPs concentration) at the pHs indicated in the inset. Note that at pHs above 5.1 the extinction at $400 \mathrm{~nm}$ is lower due to the partial reduction of the Au precursor.

Figure S3. (A-C) Additional HRTEM images and (D) histogram of branched AuNPs obtained at pH 4.1 using $106.6 \mathrm{pM}$ of seed. Inset in panel D shows a photograph of the colloidal dispersion of purified NPs.

Figure S4. (A-C) Additional HRTEM images and (D) histogram of branched AuNPs obtained at pH 4.1 using $53.3 \mathrm{pM}$ Au seeds. Inset in panel D shows a photograph of the colloidal dispersion of purified NPs.

Figure S5. (A-C) Additional HRTEM images and (D) histogram of branched AuNPs obtained at pH 4.1 using $35.4 \mathrm{pM}$ Au seeds. Inset in panel D shows a photograph of the colloidal dispersion of purified NPs.

Figure S6. (A-C) Additional HRTEM images and (D) histogram of branched AuNPs obtained at pH 4.1 using $26.7 \mathrm{pM}$ Au seeds. Inset in panel D shows a photograph of the colloidal dispersion of purified NPs.

Figure S7. (A-C) Additional HRTEM images and (D) histogram of branched AuNPs obtained at pH 4.1 using $8.9 \mathrm{pM} \mathrm{Au}$ seeds. Inset in panel D shows a photograph of the colloidal dispersion of purified NPs.

Figure S8. Normalized extinction and HRTEM images of AuNPs obtained using $26.7 \mathrm{pM} \mathrm{Au} \mathrm{seeds} \mathrm{and} \mathrm{at} \mathrm{pHs}$ 2.5 (A-C) and 4.1 (D-E).

Figure S9:Normalized extinction and HRTEM images of AuNPs obtained using $8.9 \mathrm{pM}$ Au seeds and at pHs 2.5 (A-C) and 4.1 (D-E).

Figure S10: (A) Time evolution of extinction spectra of AuNP growth at $\mathrm{pH} 2.5$ upon injection of $53.3 \mathrm{pM} \mathrm{Au}$ seeds. (B-C) Time evolution of extinction spectra of AuNP growth at pH 4.1 upon injection of $53.3 \mathrm{pM}(\mathrm{B})$ or 
$26.7 \mathrm{pM}(\mathrm{C}) \mathrm{Au}$ seeds. The spectra were recorded between 3 minutes (red line) and 240 minutes (blue line) of reaction.

Figure S11. Vis-NIR spectra of Au colloids withdraw at different reaction time as indicated (see further discussion below and experimental section for details).

Figure S12: TEM images of arrested intermediates. PEG-SH injected at 5 seconds of reaction.

Figure S13: TEM images of arrested intermediates. PEG-SH injected at 15 second (A) and 5, 30 and 90 minutes (B, C and D respectively).

Figure S14: (A and B) 3D STEM tomographic reconstruction of two different nanoparticles along two different viewing directions of the Au nanoparticles. (C and D) Two different ortho slices from A (C) and B (D) through the reconstruction revealing the location of holes and defects in the Au nanostructures (Au nanoparticle obtained at pH 4.1 and with 8.9 pM Au seeds).

Figure S15. Dynamic light scattering (DLS) analysis of PAH-Au(III) at pH 2.5 (A), 3(B), 4(C) and 4.8 (D). The data represent the mean of 5 consecutive runs performed at $22^{\circ} \mathrm{C}$ and are referred to distribution by intensity).

Figure S16. (A-C) Extinction spectra of branched AuNPs dispersed in different water-glycerol mixtures, as indicated, with the LSPR centered at $530 \mathrm{~nm}, 600 \mathrm{~nm}$ and $709 \mathrm{~nm}$ respectively. (D-F). First-order derivates of the AuNPs dispersed in different water-glycerol mixtures with the LSPR centered at $530 \mathrm{~nm}, 600 \mathrm{~nm}$ and 709 $\mathrm{nm}$ respectively. (G-I) Second-order derivates of the AuNPs dispersed in different water-glycerol with the LSPR centered at $530 \mathrm{~nm}, 600 \mathrm{~nm}$ and $709 \mathrm{~nm}$ respectively.

Figure S17. Inflection point method for three branched AuNPs dispersed in different water-glycerol mixtures, as indicated, with the LSPR centered at $530 \mathrm{~nm}, 600 \mathrm{~nm}$ and $709 \mathrm{~nm}$ respectively. (A, C, D) $1^{\text {st }} \mathrm{IF}$, maximum LSPR position and $2^{\text {nd }}$ IF plotted against the different local refractive indexes for AuNPs with the LSPR centerd at $530 \mathrm{~nm}, 600 \mathrm{~nm}$ and $709 \mathrm{~nm}$ respectively. (B, D, F) Sensitivity of local refractive index media on peak shifts $1^{\text {st }} I F$, maximum LSPR position and $2^{\text {nd }} I F$ for AuNPs with the LSPR centered at $530 \mathrm{~nm}, 600 \mathrm{~nm}$ and $709 \mathrm{~nm}$ respectively.

Figure 18. (A) Normalized Vis-NIR spectra and (B) z-potential of AuNPs@PAH and post-functionalized with PAcMA (denoted as AuNPs@COOH). (C-D) TEM images of AuNPs@COOH.

Figure S19. FTIR spectra of PAcMA vs AuNPs@PAH@PAcMA. 
Figure S20. Comparative normalized NIR-Vis spectra of AuNPs@PAH@PacMA after purification using PBS $\mathrm{pH}=7.4(([\mathrm{Phosphate}]=100 \mathrm{mM}$ and $[\mathrm{NaCl}]=200 \mathrm{mM})$ obtained with or without activation of $\mathrm{COOH}$ via EDC/NHS. 


\section{2.- Movies}

Movie S1. Ortho slices through the reconstruction of the particles made with $53.3 \mathrm{pM}$ of gold seed solution.

Movie S2. Ortho slices through the reconstruction of the particles made with $8.9 \mathrm{pM}$ of gold seed solution.

Movie S3. Rotation of the 3D visualization of the particles made with $53.3 \mathrm{pM}$ of gold seed solution.

Movie S4. Rotation of the 3D visualization of the particles made with $8.9 \mathrm{pM}$ of gold seed solution.

\section{3.- Tables}

Table S1. pH, diameter and polydispersity index (PDI) of PAH-Au(III) solutions.

\section{4.- References}



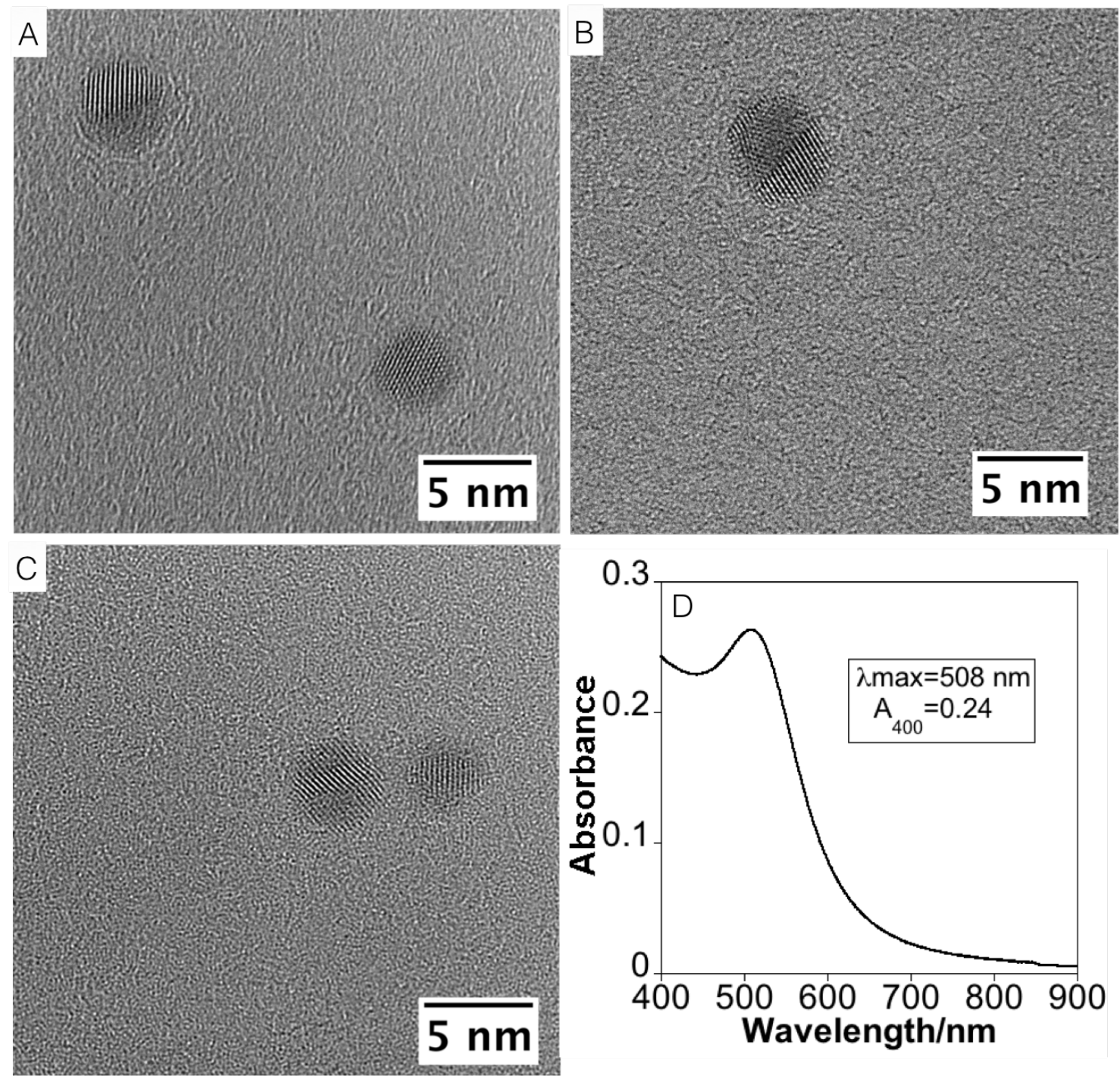

Figure S1. (A-C) HRTEM images of Au seeds after aging for $1 \mathrm{~h}$ at $40{ }^{\circ} \mathrm{C}$. (D) Vis-NIR spectrum of AuNPs seeds in water. 


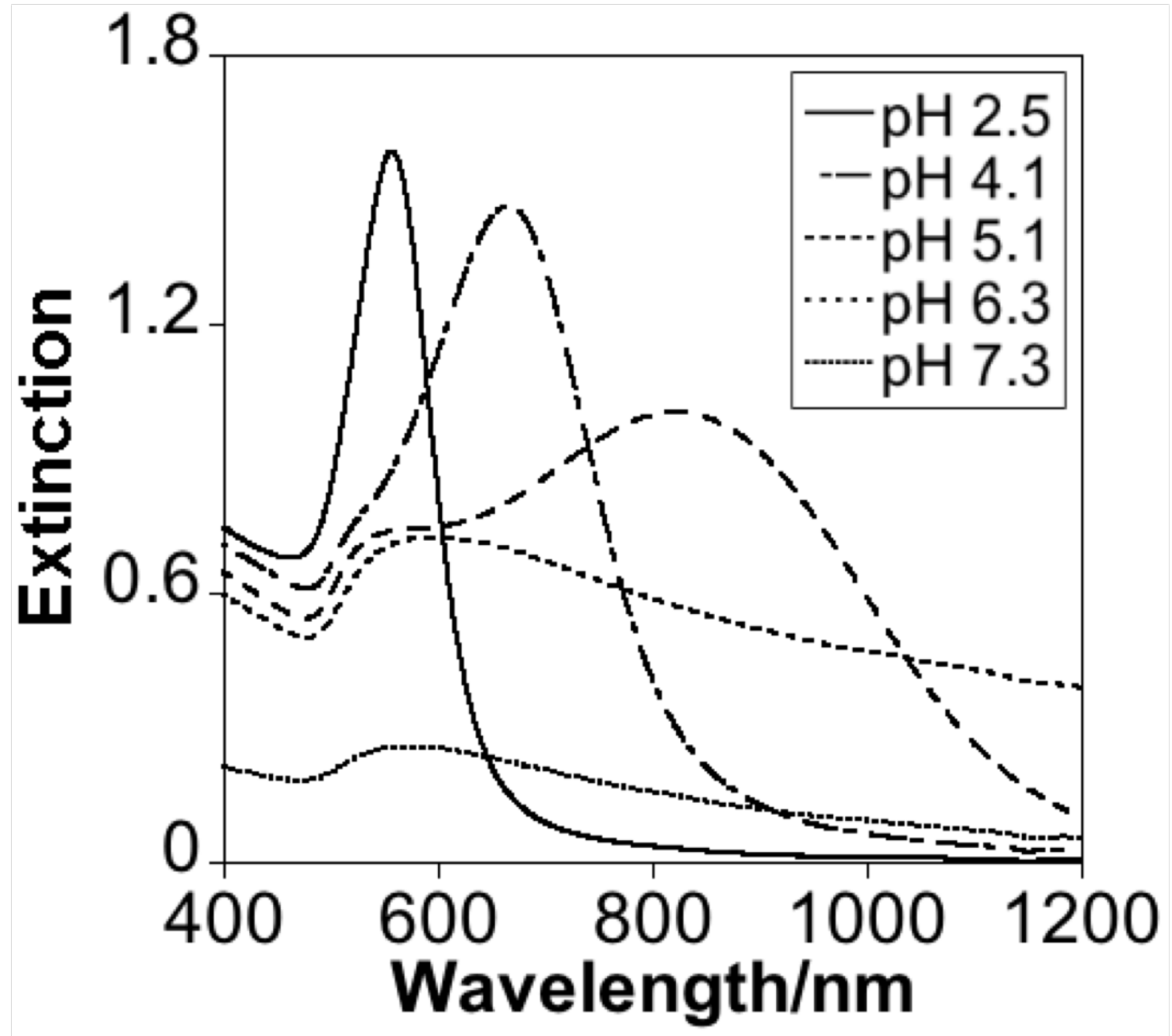

Figure S2. Vis-NIR spectra of AuNPs obtained adding $53.3 \mathrm{pM}$ seeds (in terms of NPs concentration) at the pHs indicated in the inset. Note that at pHs above 5.1 the extinction at $400 \mathrm{~nm}$ is lower due to the partial reduction of the Au precursor. 

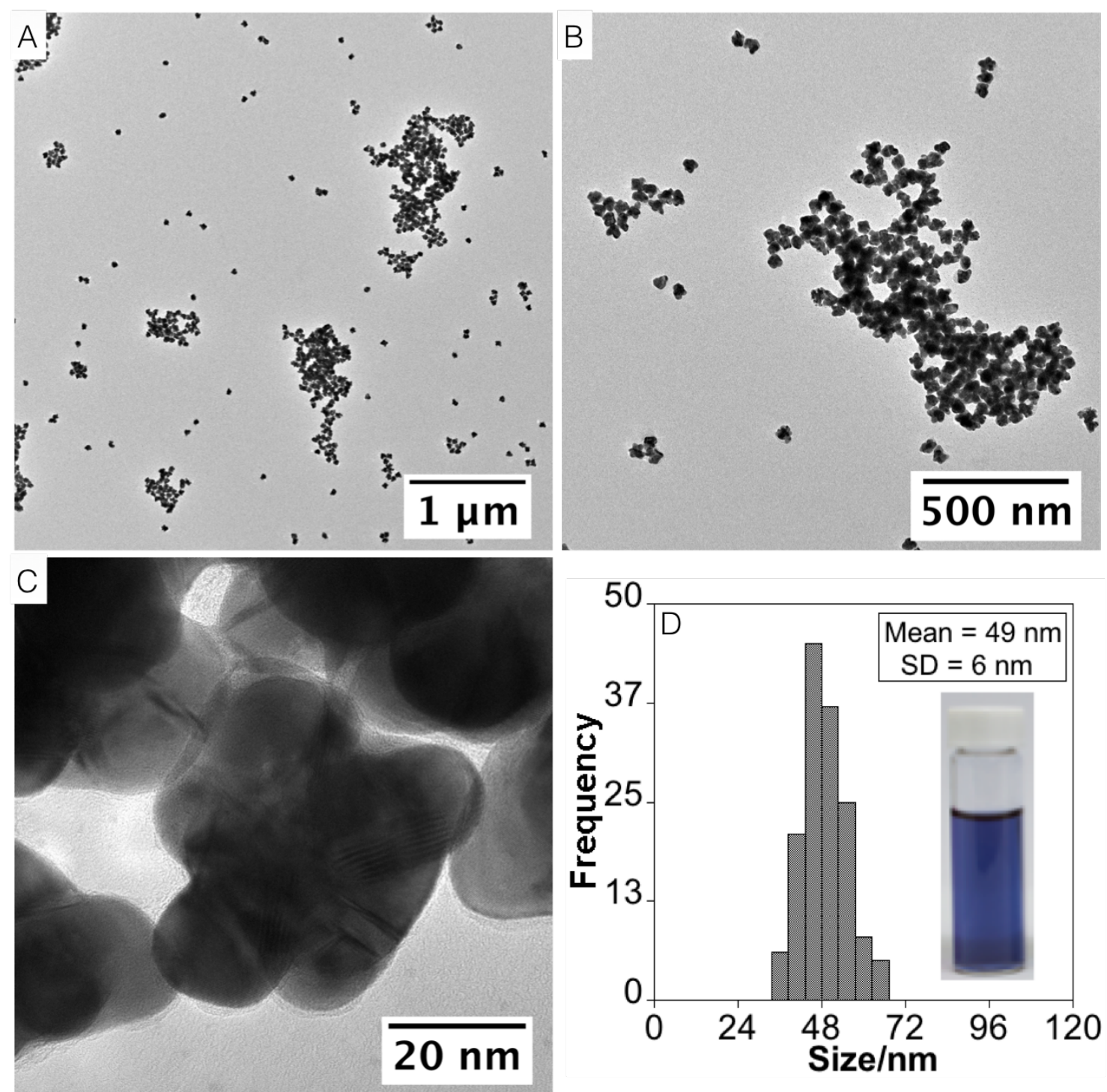

Figure S3. (A-C) Additional HRTEM images and (D) histogram of branched AuNPs obtained at pH 4.1 using $106.6 \mathrm{pM}$ of seed. Inset in panel D shows a photograph of the colloidal dispersion of purified NPs. 


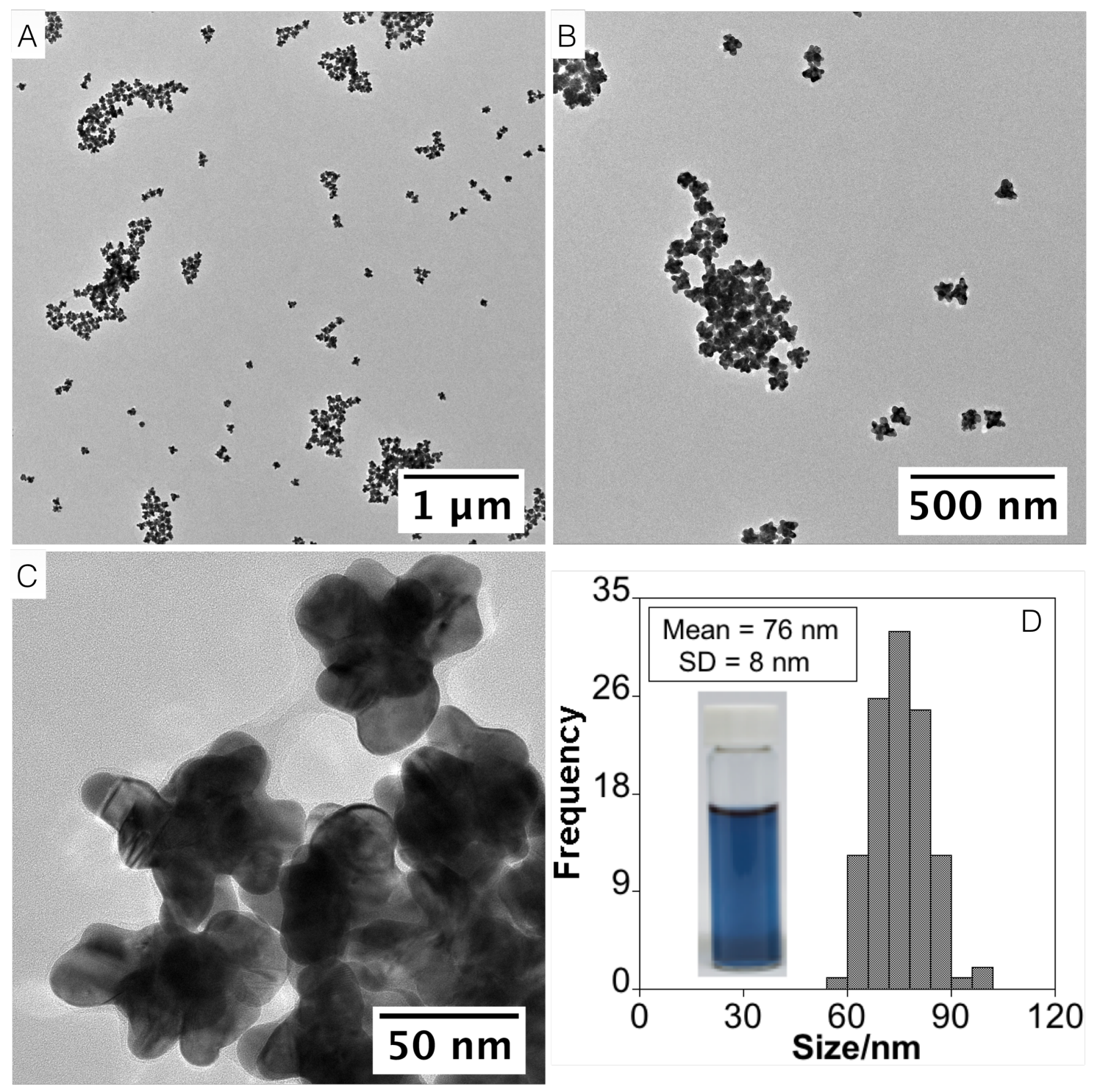

Figure S4. (A-C) Additional HRTEM images and (D) histogram of branched AuNPs obtained at pH 4.1 using $53.3 \mathrm{pM}$ Au seeds. Inset in panel D shows a photograph of the colloidal dispersion of purified NPs. 


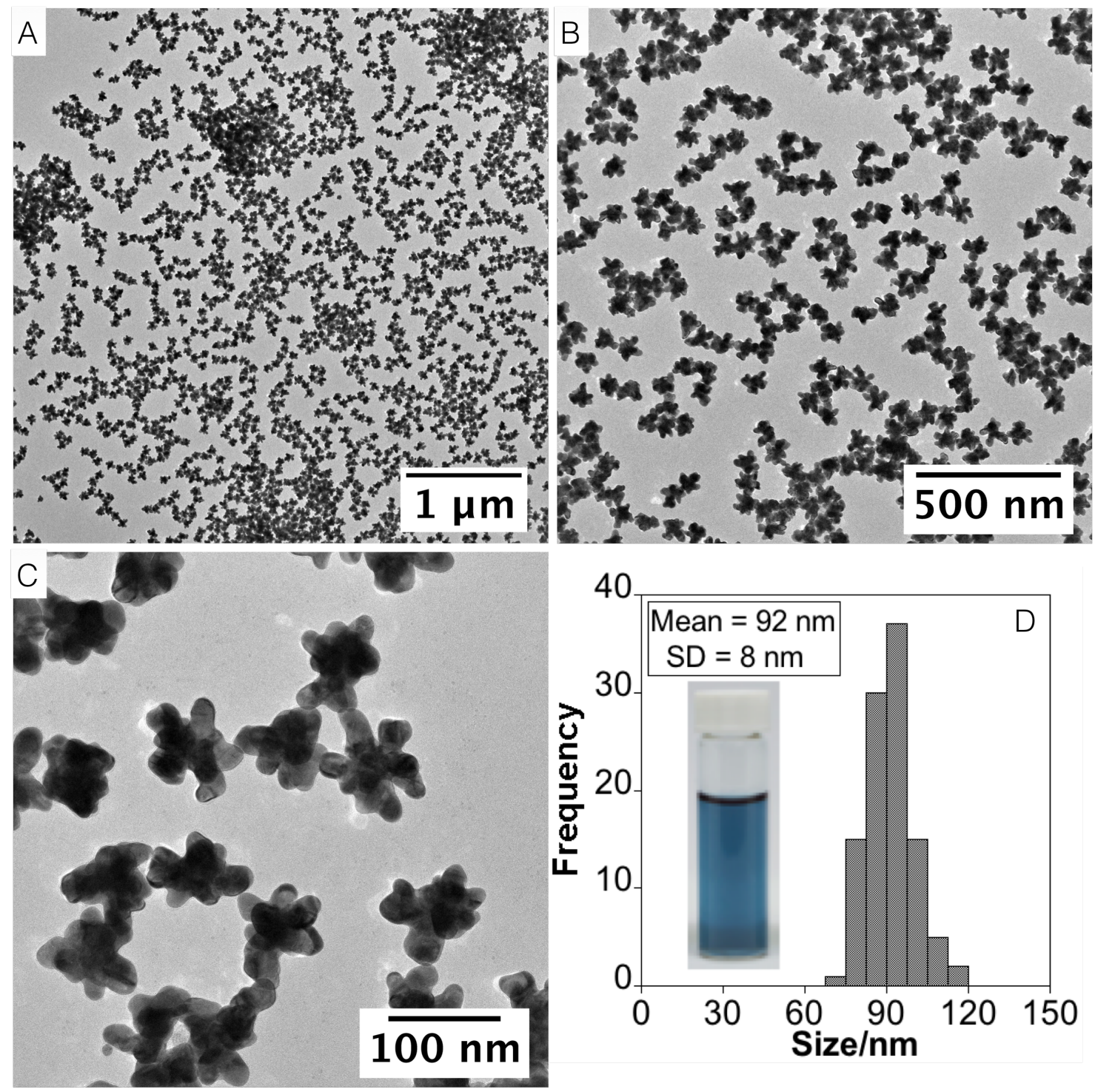

Figure S5. (A-C) Additional HRTEM images and (D) histogram of branched AuNPs obtained at pH 4.1 using $35.4 \mathrm{pM}$ Au seeds. Inset in panel D shows a photograph of the colloidal dispersion of purified NPs. 


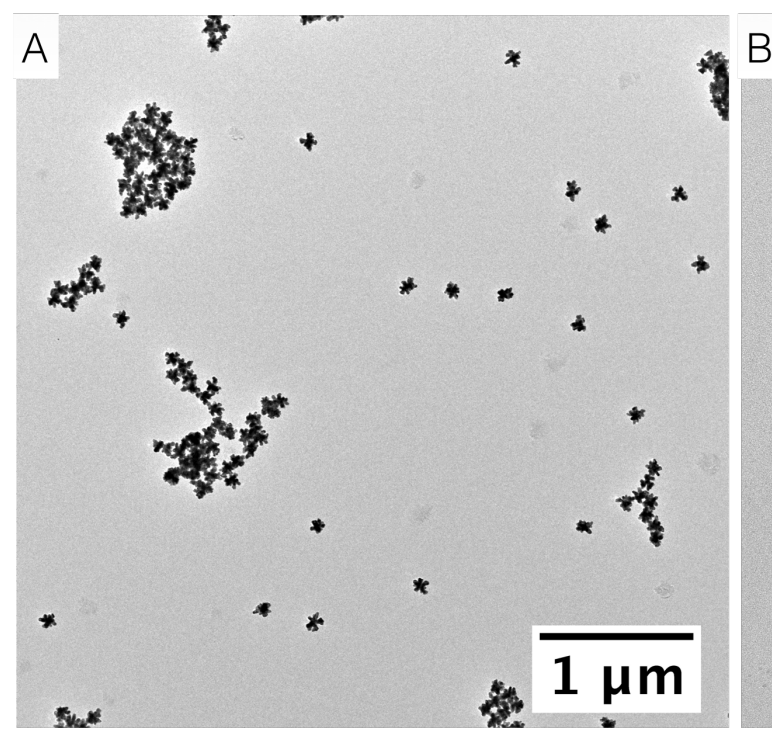

$100 \mathrm{~nm}$
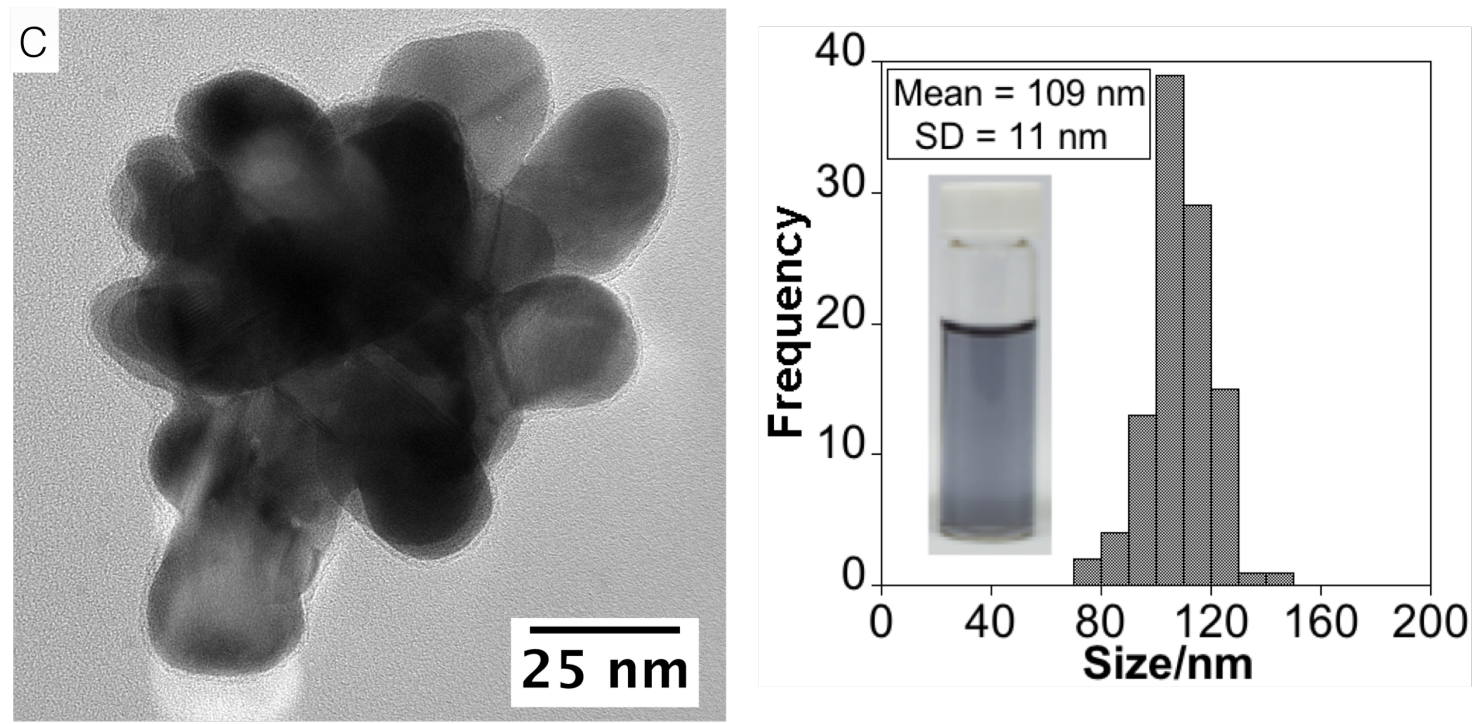

Figure S6. (A-C) Additional HRTEM images and (D) histogram of branched AuNPs obtained at pH 4.1 using $26.7 \mathrm{pM}$ Au seeds. Inset in panel D shows a photograph of the colloidal dispersion of purified NPs. 

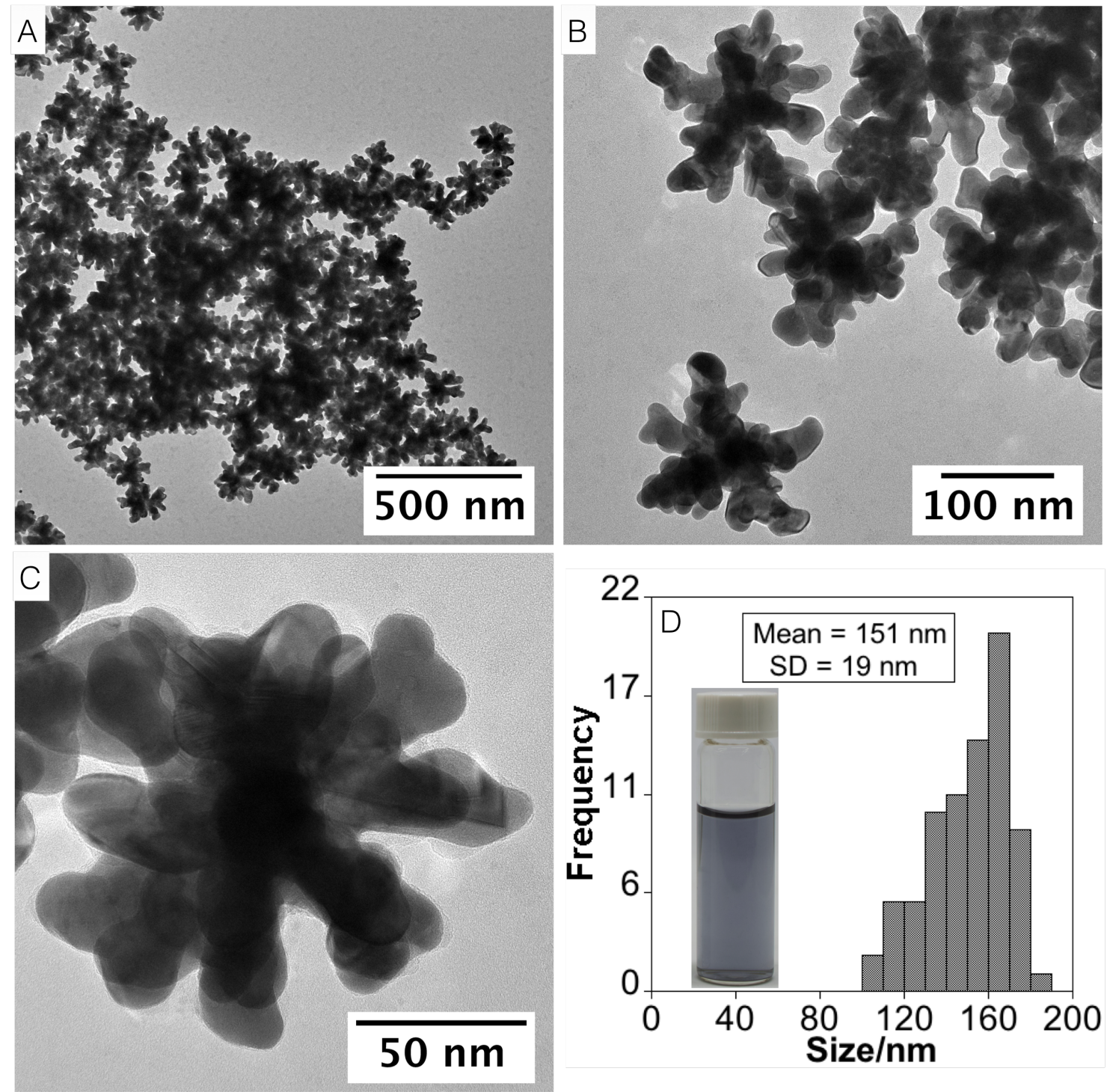

Figure S7. (A-C) Additional HRTEM images and (D) histogram of branched AuNPs obtained at pH 4.1 using $8.9 \mathrm{pM}$ Au seeds. Inset in panel D shows a photograph of the colloidal dispersion of purified NPs. 

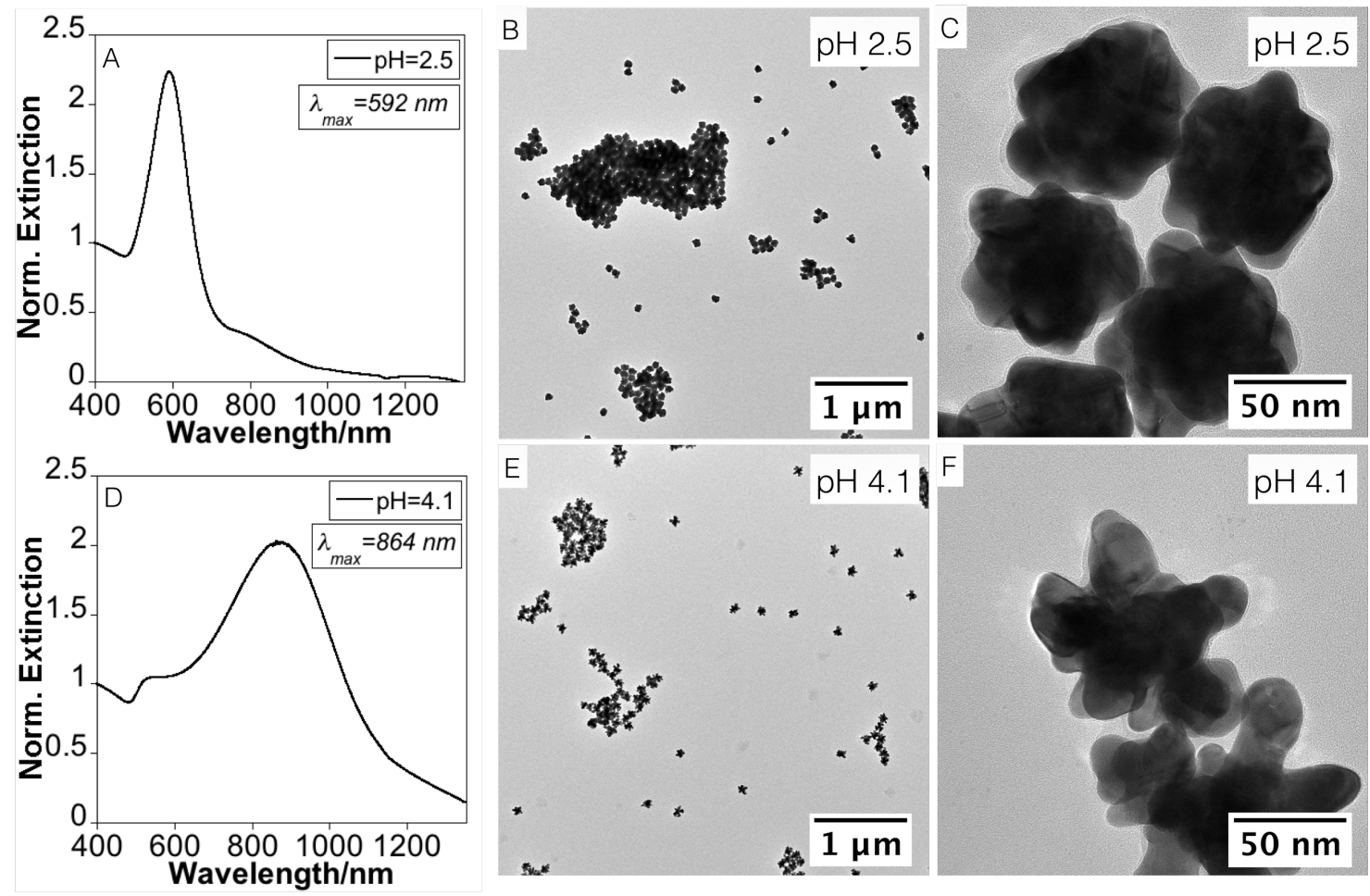

Figure S8. Normalized extinction and HRTEM images of AuNPs obtained using $26.7 \mathrm{pM}$ Au seeds and at pHs 2.5 (A-C) and 4.1 (D-E).
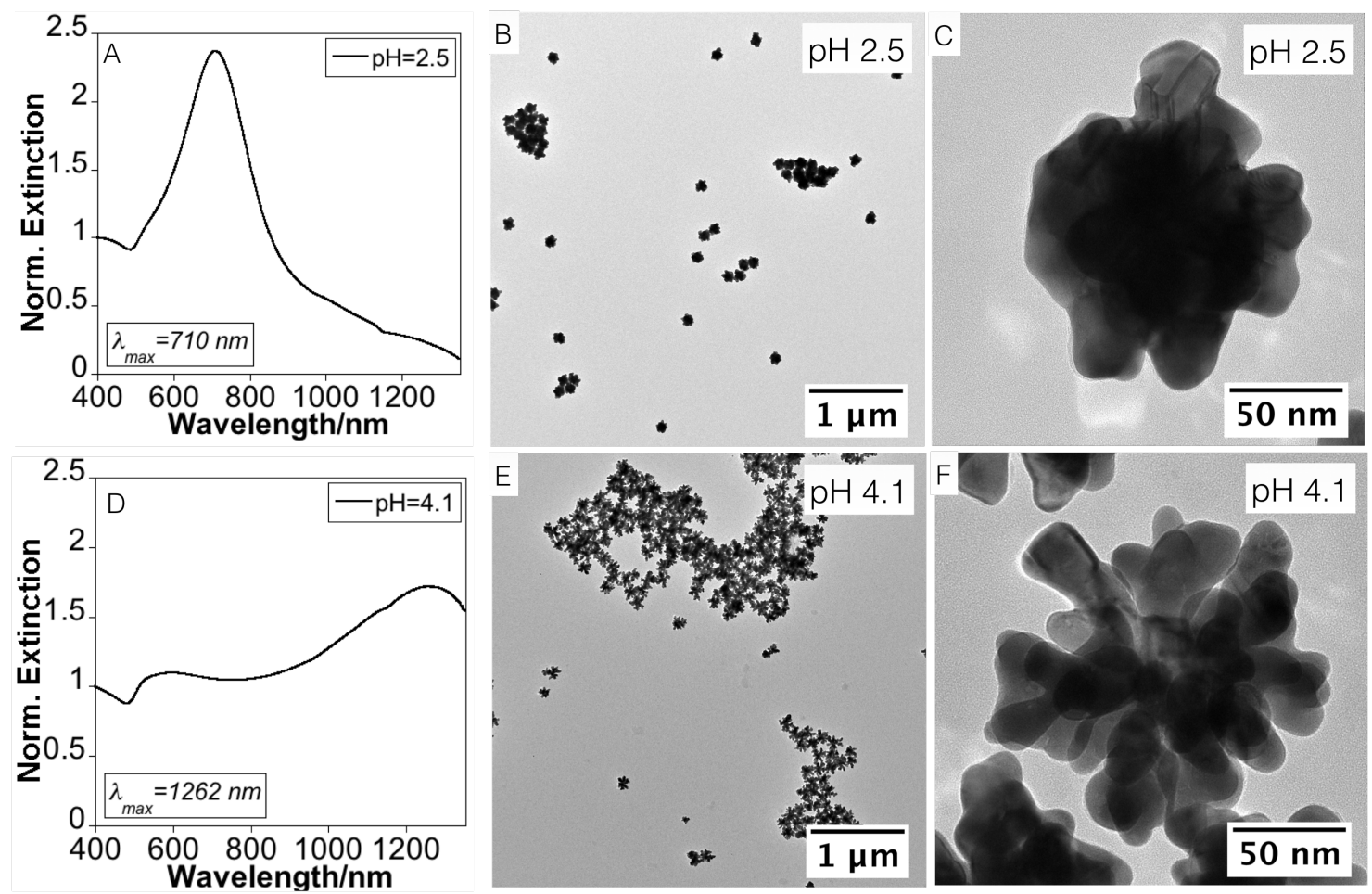
Figure S9:Normalized extinction and HRTEM images of AuNPs obtained using $8.9 \mathrm{pM}$ Au seeds and at pHs 2.5 (A-C) and 4.1 (D-E). 

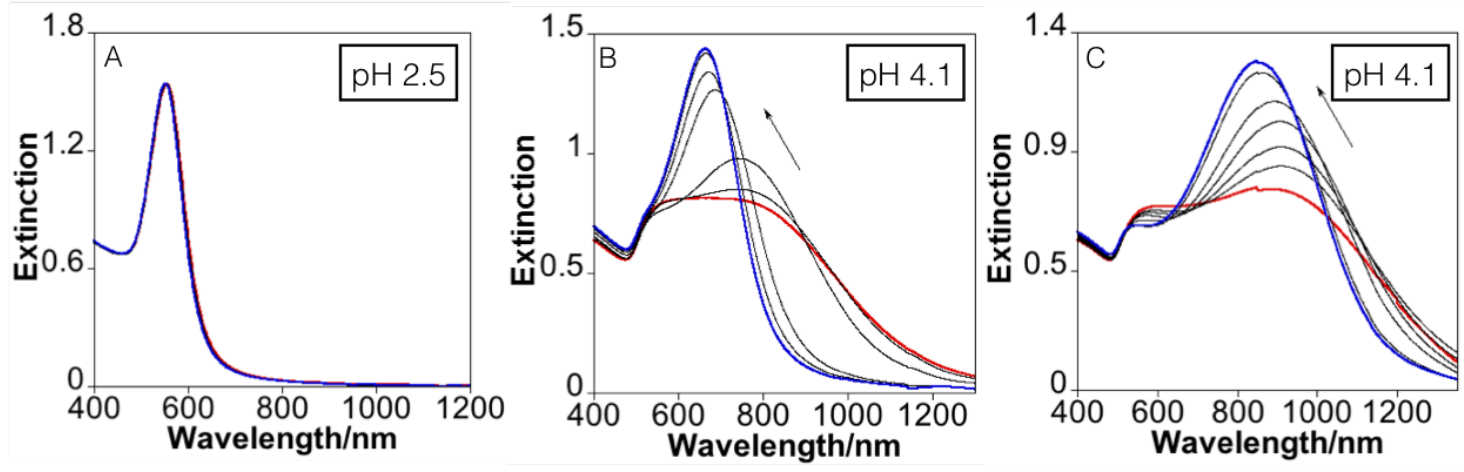

Figure S10: (A) Time evolution of extinction spectra of AuNP growth at $\mathrm{pH} 2.5$ upon injection of $53.3 \mathrm{pM} \mathrm{Au}$ seeds. (B-C) Time evolution of extinction spectra of AuNP growth at $\mathrm{pH} 4.1$ upon injection of $53.3 \mathrm{pM}$ (B) or $26.7 \mathrm{pM}(\mathrm{C})$ Au seeds. The spectra were recorded between 3 minutes (red line) and 240 minutes (blue line) of reaction. 


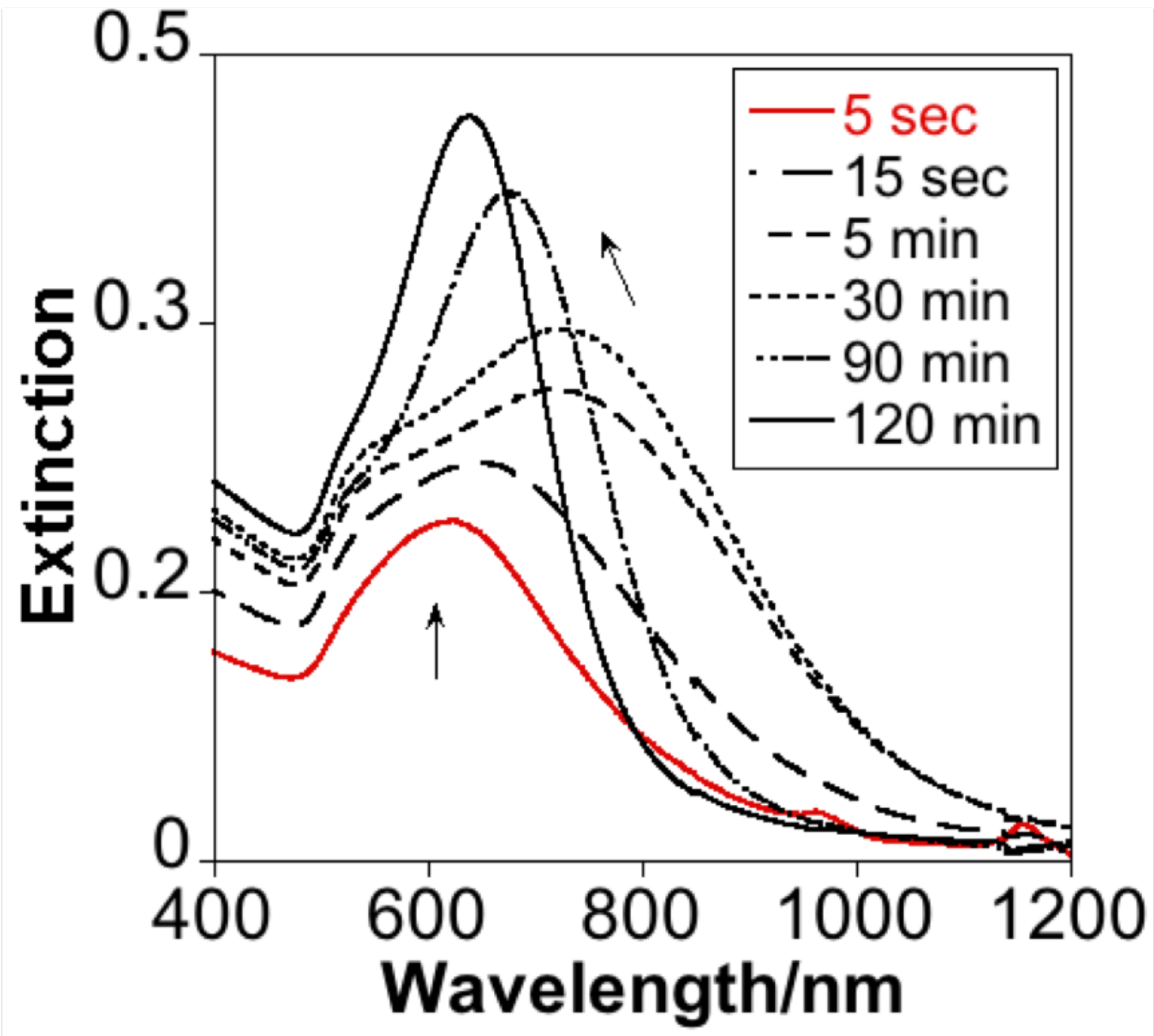

Figure S11. Vis-NIR spectra of Au colloids withdraw at different reaction time as indicated (see further discussion below and experimental section for details).

The growth of the AuNPs was arrested at different reaction times by adding PEG-SH in excess in the reaction medium (see experimental part). To remove the excess of thiolated molecules and unreacted gold salt, the intermediates were washed by centrifugation and analyzed by Vis-NIR spectroscopy and electron microscopy. Interestingly, the optical properties of the different intermediates before and after the arresting/purification step are similar (Figure 3A and S11) indicating that the morphology of the particles was preserved. Similar results were also observed when comparing the time-course of the arrested reaction intermediates with that of the in situ reaction shown in Figure S10B. 


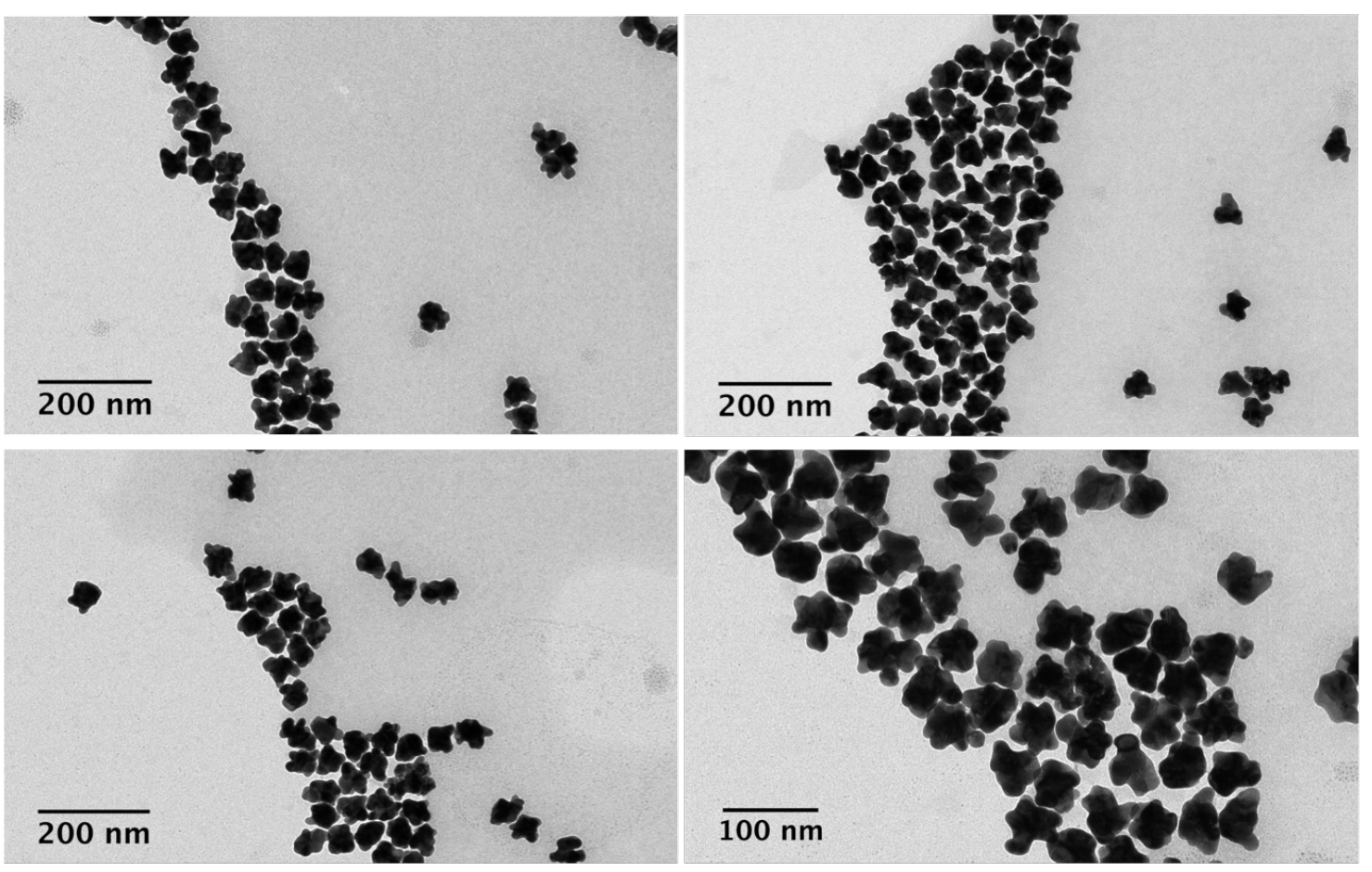

Figure S12: TEM images of arrested intermediates. PEG-SH injected at 5 seconds of reaction. 


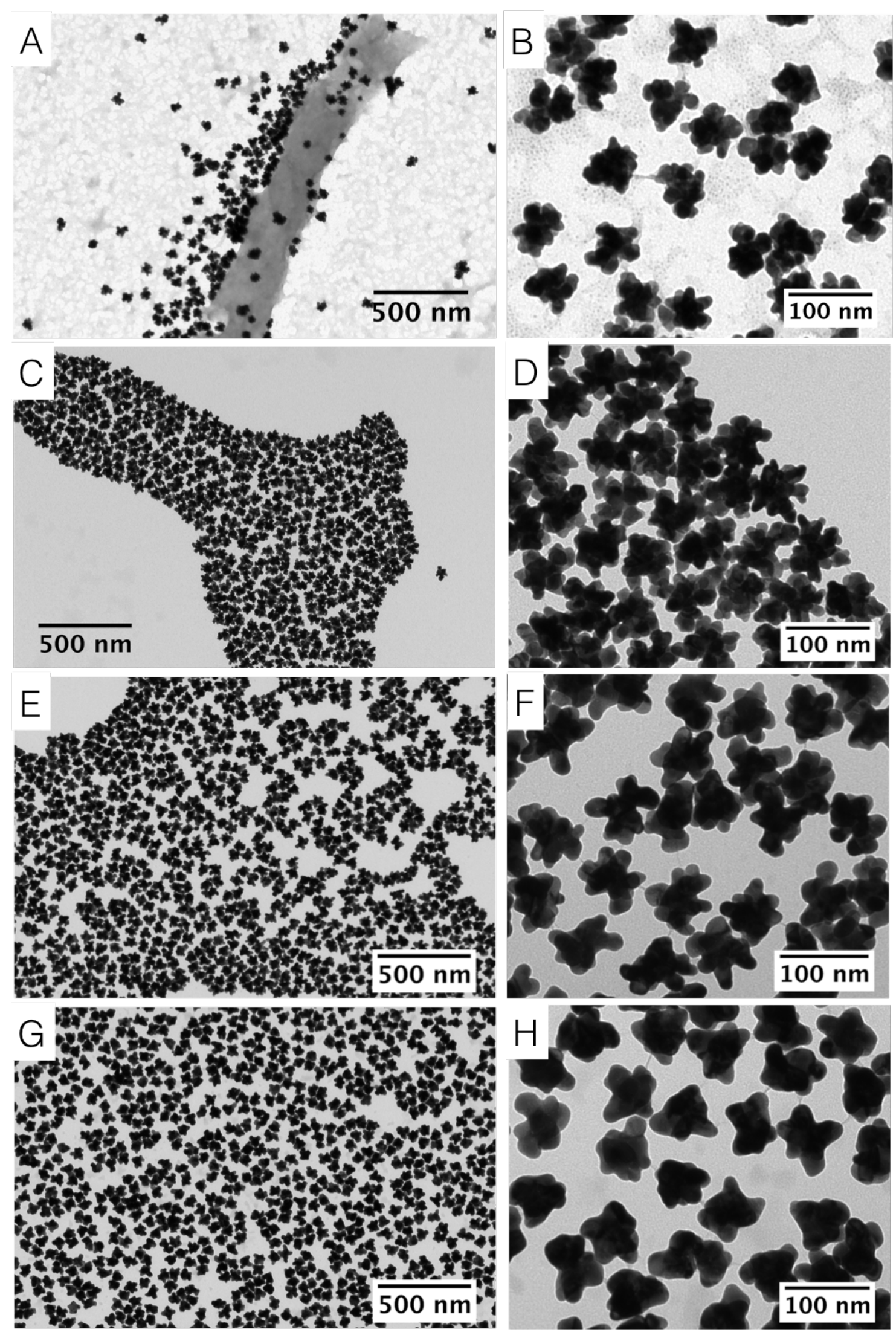


Figure S13. TEM images of arrested intermediates. PEG-SH injected at 15 second (A) and 5, 30 and 90 minutes ( $B, C$ and $D$ respectively).
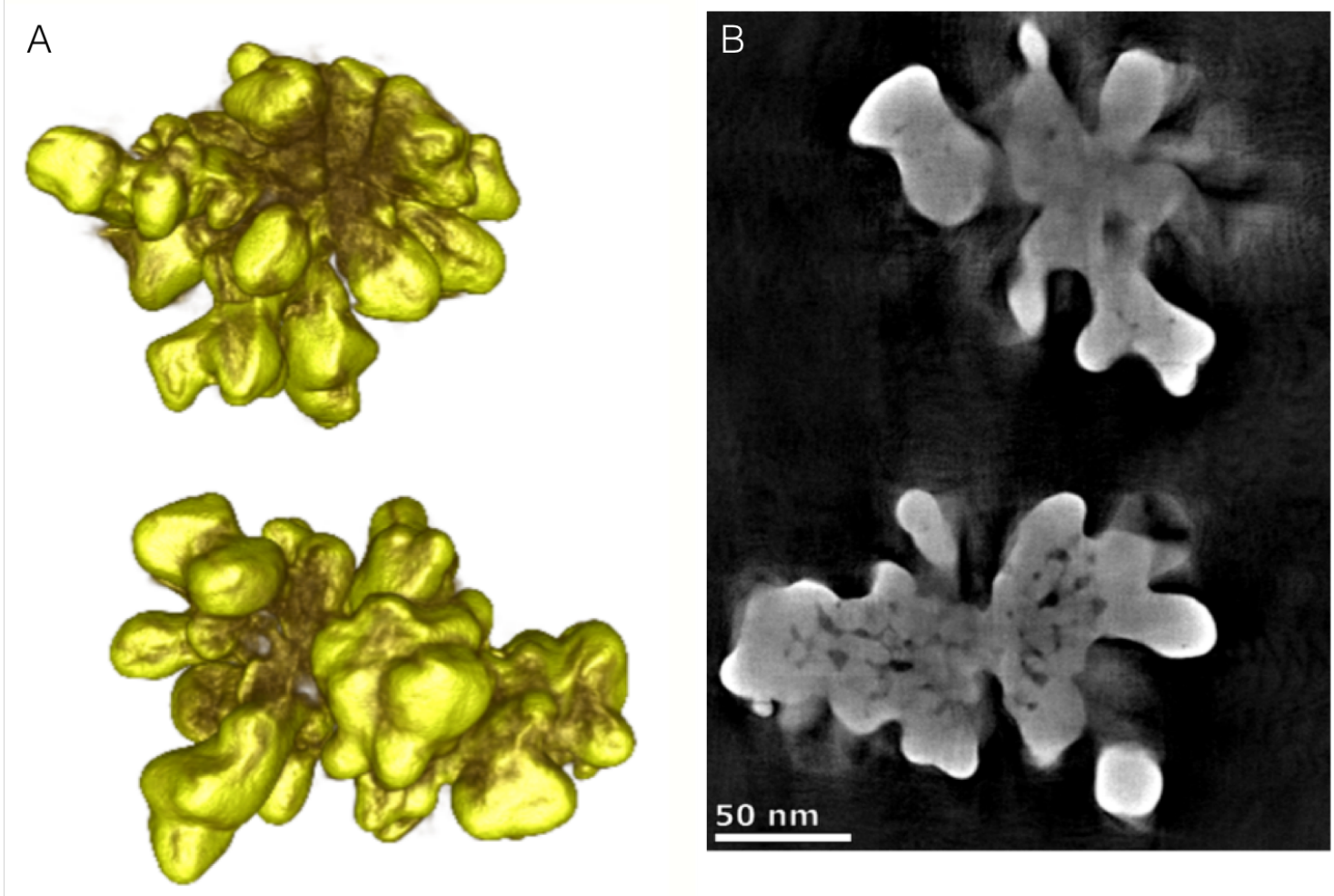

Figure S14. (A and B) 3D STEM tomographic reconstruction of two different nanoparticles along two different viewing directions of the Au nanoparticles. ( $C$ and $D)$ Two different ortho slices from $A(C)$ and $B(D)$ through the reconstruction revealing the location of holes and defects in the Au nanostructures (Au nanoparticle obtained at $p H 4.1$ and with 8.9 pM Au seeds). 


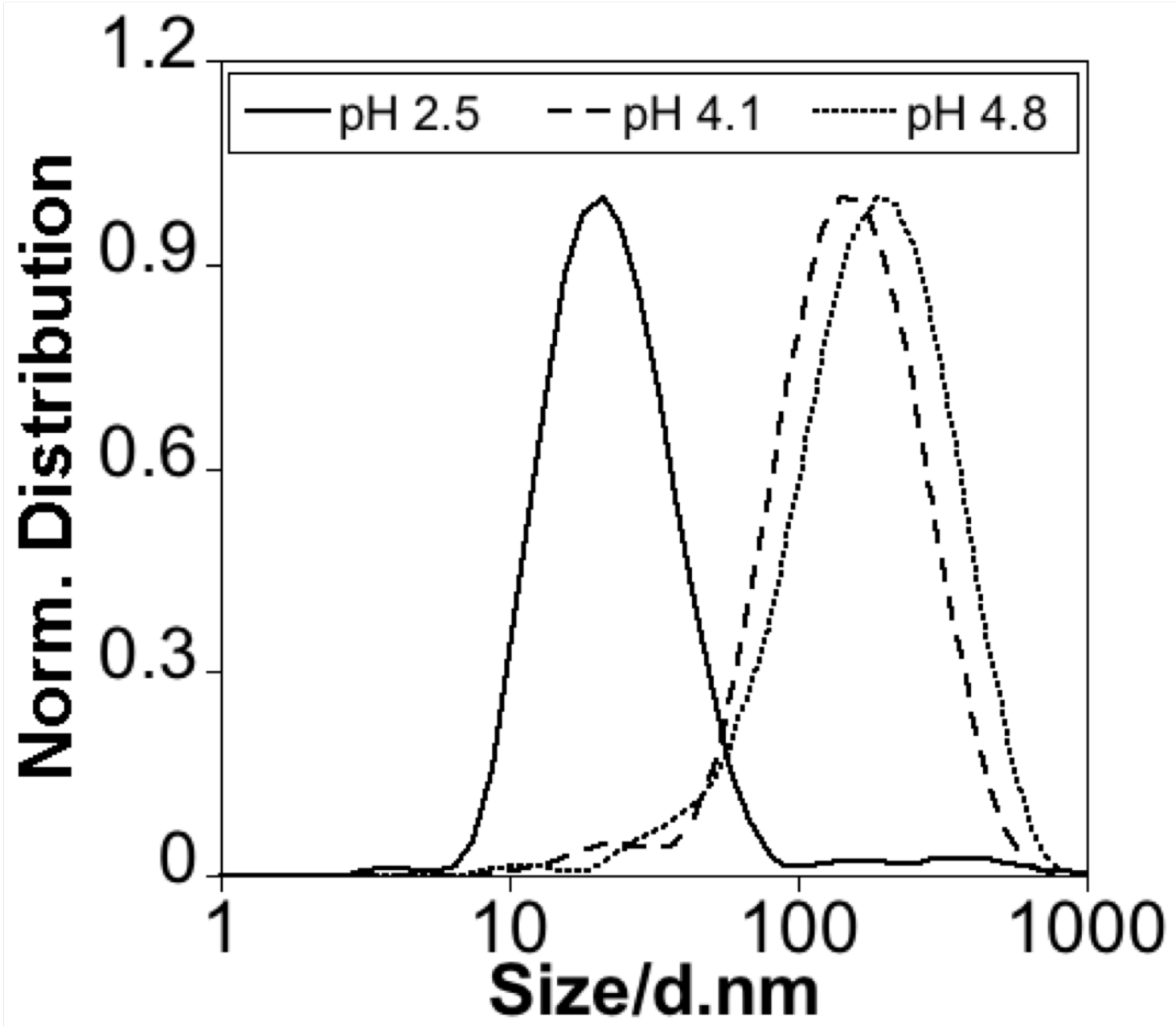

Figure S15. Dynamic light scattering (DLS) analysis of PAH-Au(III) at pH $2.5(A), 3(B), 4(C)$ and $4.8(D)$. The data represent the mean of 5 consecutive runs performed at $22^{\circ} \mathrm{C}$ and are referred to distribution by intensity). 

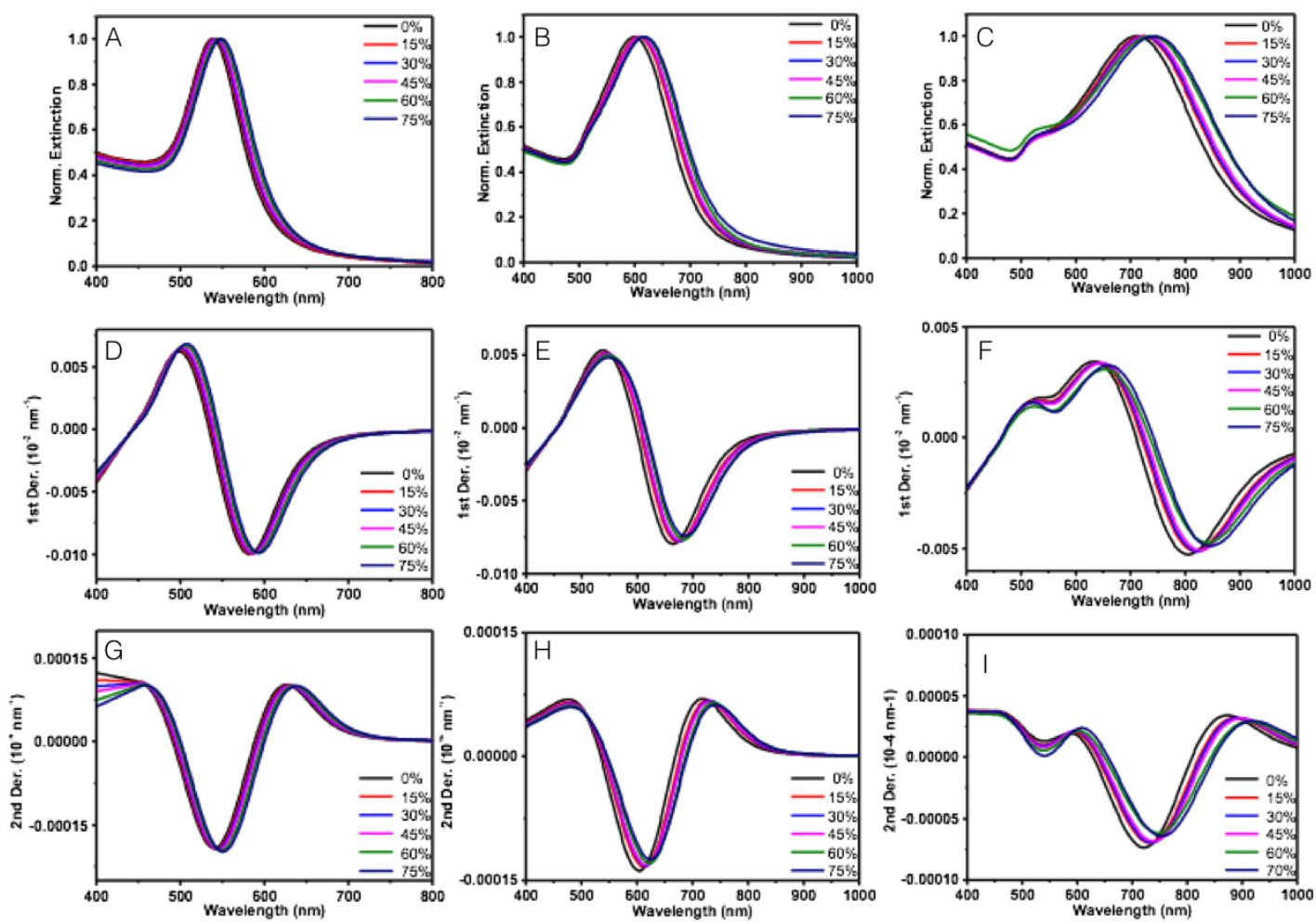

Figure S16. (A-C) Extinction spectra of branched AuNPs dispersed in different water-glycerol mixtures, as indicated, with the LSPR centered at $530 \mathrm{~nm}, 600 \mathrm{~nm}$ and $709 \mathrm{~nm}$ respectively. (D-F). First-order derivates of the AuNPs dispersed in different water-glycerol mixtures with the LSPR centered at $530 \mathrm{~nm}, 600 \mathrm{~nm}$ and 709 $n m$ respectively. (G-I) Second-order derivates of the AuNPs dispersed in different water-glycerol with the LSPR centered at $530 \mathrm{~nm}, 600 \mathrm{~nm}$ and $709 \mathrm{~nm}$ respectively.

The refractive index of the water-glycerol mixtures was calculated through the Lorentz-Lorentz equation (see SI, Figure SX) , as follows:

$$
\frac{n_{w g}^{2}-1}{n_{w g}^{2}+2}=\varphi_{w} \frac{n_{w}^{2}-1}{n_{w}^{2}+2}+\varphi_{g} \frac{n_{g}^{2}-1}{n_{g}^{2}+2}
$$

Where $\mathrm{n}_{\mathrm{wg}}$ is the refractive index of the mixture, $\mathrm{n}_{\mathrm{w}}(1.33)$ and $\mathrm{n}_{\mathrm{g}}(1.47)$ are the refractive index of the water and glycerol, respectively, and $\phi_{\mathrm{w}}$ and $\phi_{\mathrm{g}}$ are their corresponding volume fractions. 

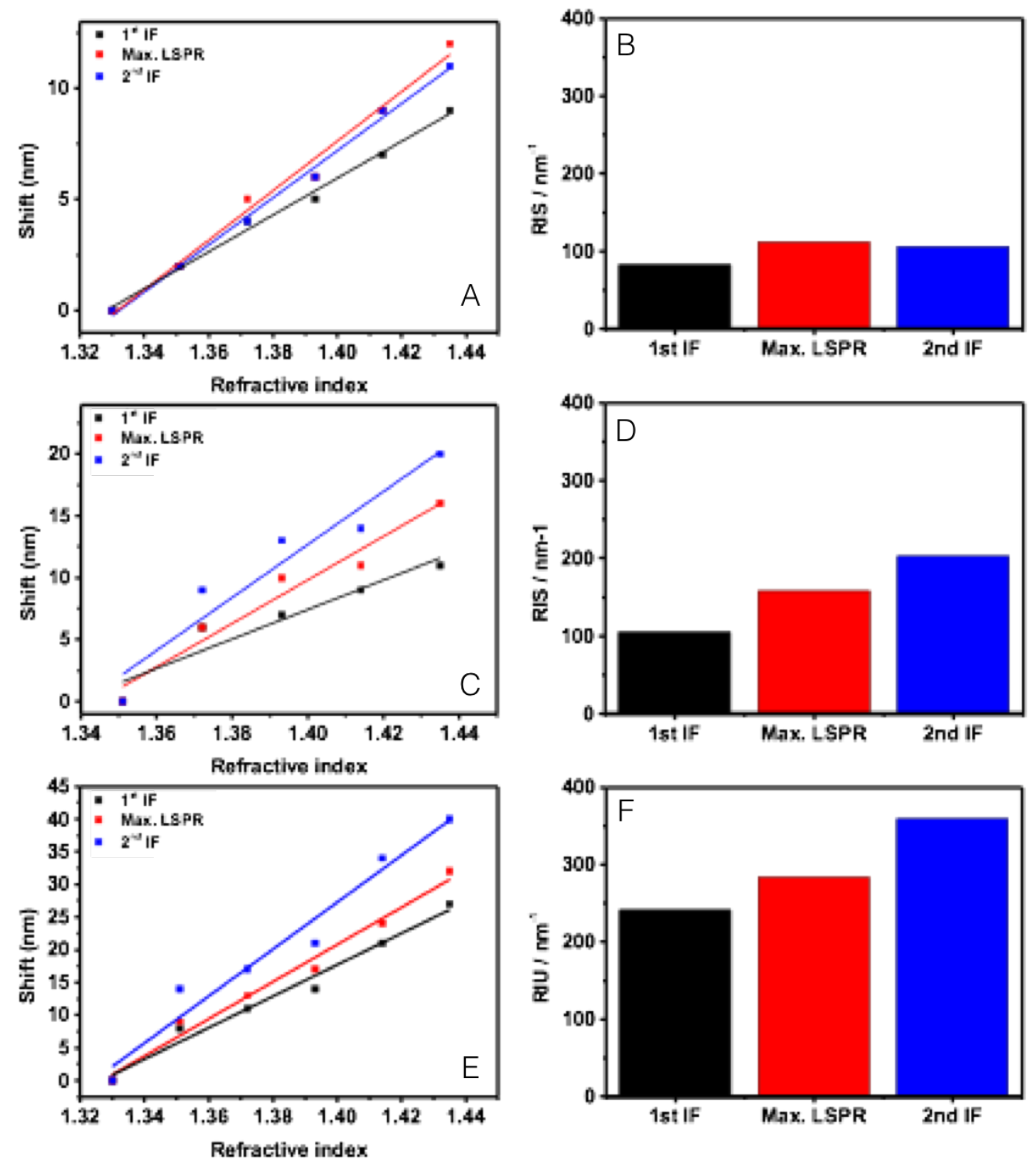

Figure S17. Inflection point method for three branched AuNPs dispersed in different water-glycerol mixtures, as indicated, with the LSPR centered at $530 \mathrm{~nm}, 600 \mathrm{~nm}$ and $709 \mathrm{~nm}$ respectively. (A, C, D) $1^{\text {st }} \mathrm{IF}$, maximum LSPR position and $2^{\text {nd }}$ IF plotted against the different local refractive indexes for AuNPs with the LSPR centerd at $530 \mathrm{~nm}, 600 \mathrm{~nm}$ and $709 \mathrm{~nm}$ respectively. $(B, D, F)$ Sensitivity of local refractive index media on peak shifts $I^{\text {st }} I F$, maximum LSPR position and $2^{\text {nd }}$ IF for AuNPs with the LSPR centered at $530 \mathrm{~nm}, 600 \mathrm{~nm}$ and $709 \mathrm{~nm}$ respectively. 

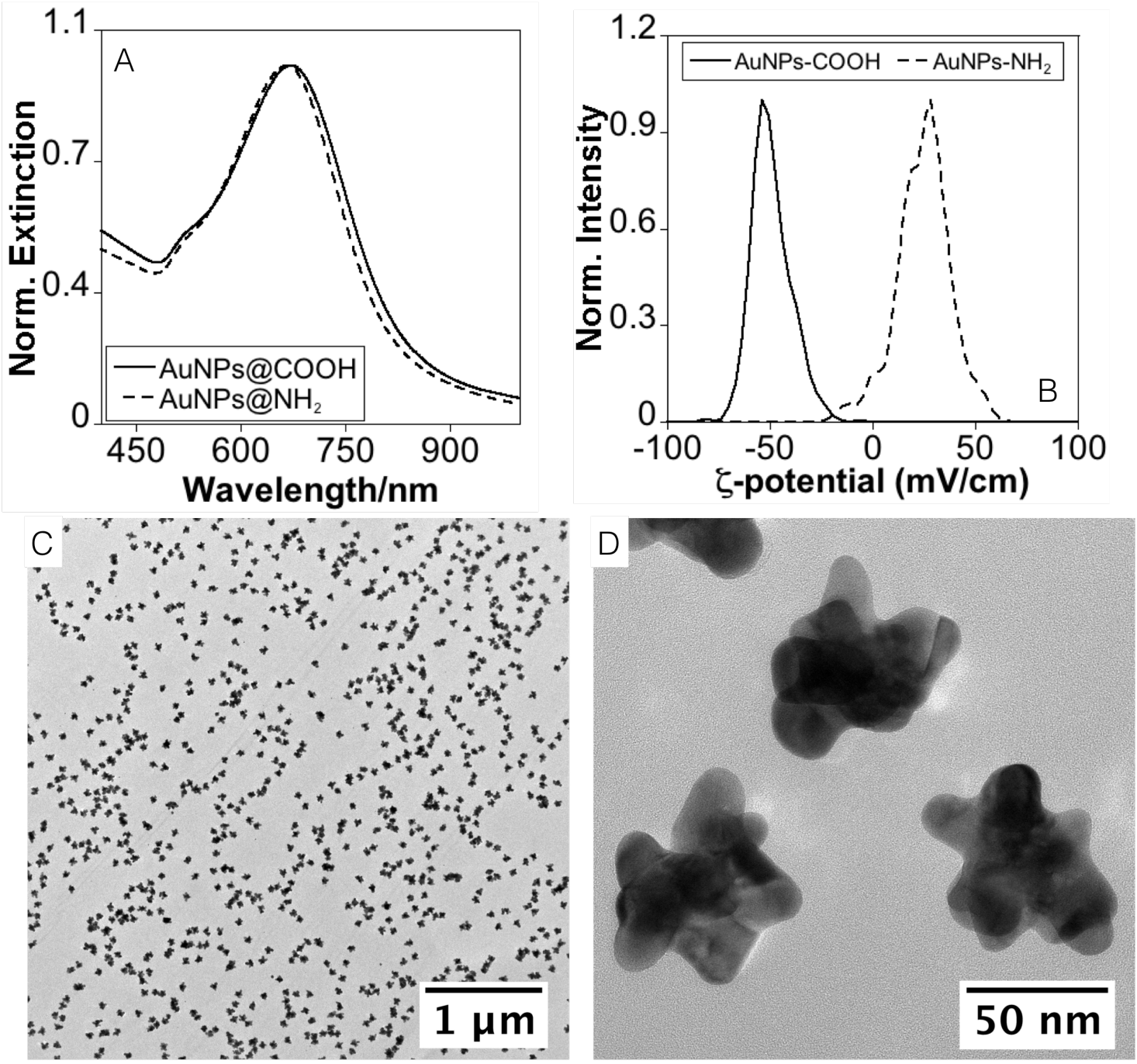

Figure 18. (A) Normalized Vis-NIR spectra and (B) z-potential of AuNPs@PAH and post-functionalized with PAcMA (denoted as AuNPs@COOH). (C-D)TEM images of AuNPs@COOH. 


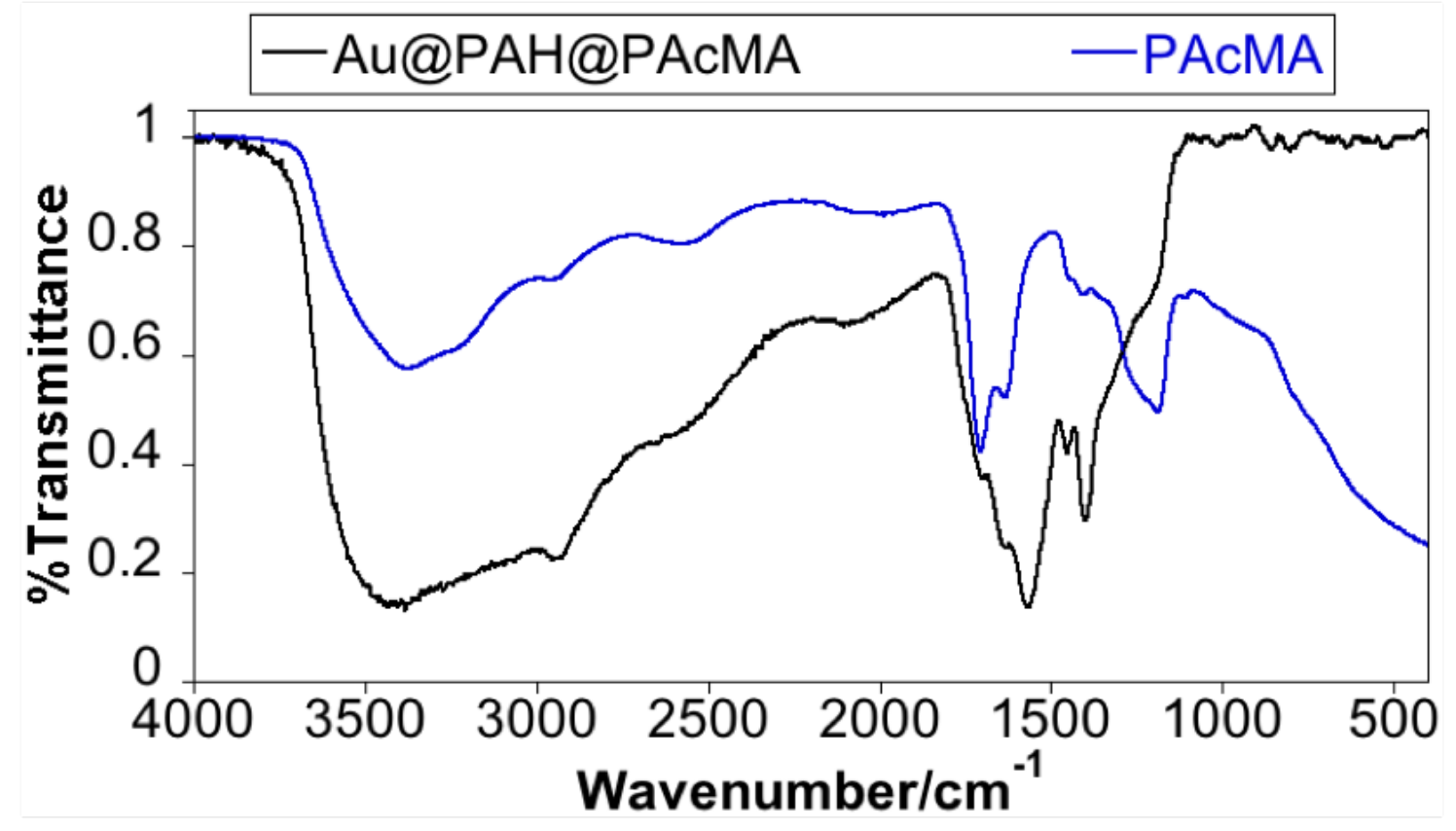

Figure S19. FTIR spectra of PAcMAvsAuNPs@PAH@PAcMA.

The FTIR spectrum of pure PAcMA shows different characteristic signals: at 3387 and $2950 \mathrm{~cm}^{-1}$ assigned to the $\mathrm{O}-\mathrm{H}$ and $\mathrm{C}-\mathrm{H}$ stretching, respectively, at 1703 and $1635 \mathrm{~cm}^{-1}$ assigned to the $\mathrm{COOH}$ stretching and at 1443 and $1408 \mathrm{~cm}^{-1}$ attributed to the $\mathrm{C}=\mathrm{O}$ stretching and $\mathrm{O}-\mathrm{H}$ bending. ${ }^{[4]}$ On the other hand, the FTIR spectrum of the AuNPs@PAH@PAcMA NPs shows the characteristic signals that could be assigned to the carboxylic moieties (COOH stretching and at 1443 and $1408 \mathrm{~cm}^{-1}$ ) as well as the band at $1560 \mathrm{~cm}^{-1}$ that could be assigned to the amide resulting of the amidation between PAH and PAcMA. ${ }^{[5]}$ 


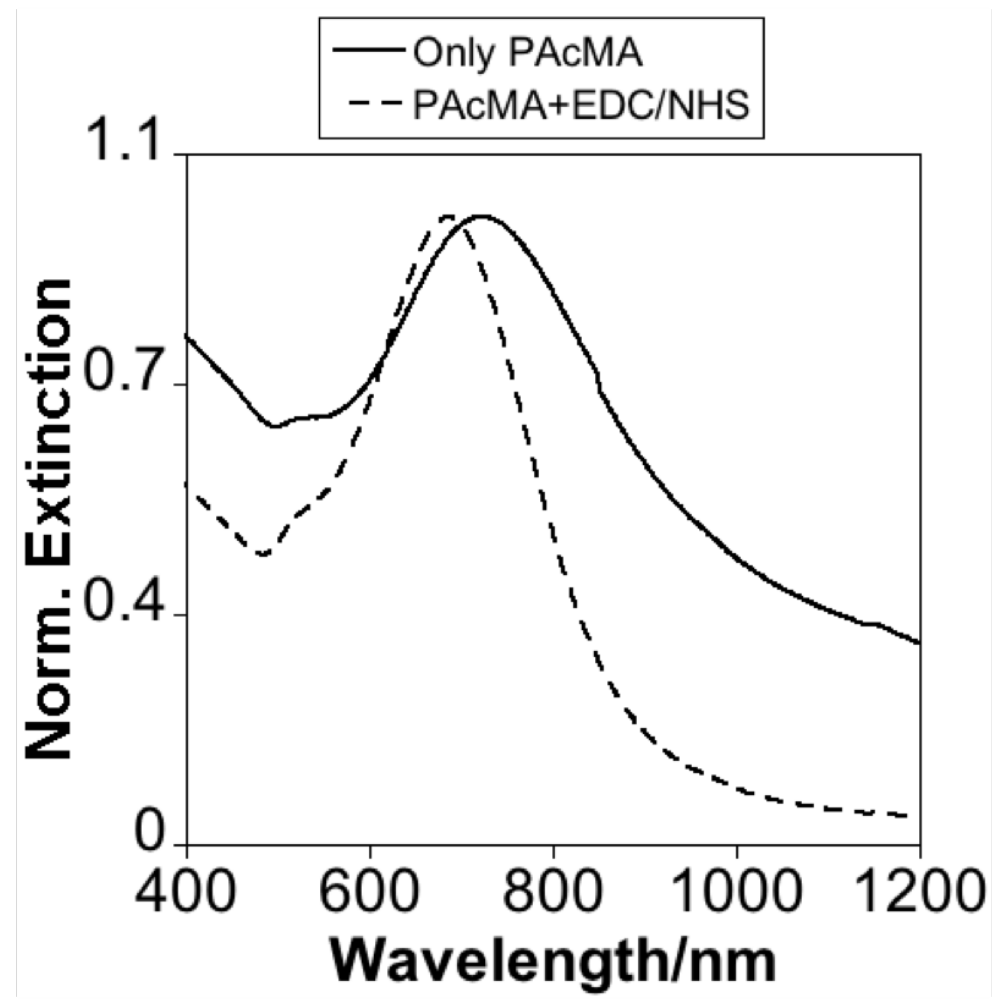

Figure S20. Comparative normalized NIR-Vis spectra of AuNPs@PAH@PacMA after purification using PBS $\mathrm{pH}=7.4(([\mathrm{Phosphate}]=100 \mathrm{mM}$ and $[\mathrm{NaCl}]=200 \mathrm{mM})$ obtained with or without activation of $\mathrm{COOH}$ via EDC/NHS.

Movie S1. Ortho slices through the reconstruction of the particles made with $53.3 \mathrm{pM}$ of gold seed solution.

Movie S2. Ortho slices through the reconstruction of the particles made with $8.9 \mathrm{pM}$ of gold seed solution.

Movie S3. Rotation of the 3D visualization of the particles made with $53.3 \mathrm{pM}$ of gold seed solution.

Movie S4. Rotation of the 3D visualization of the particles made with $8.9 \mathrm{pM}$ of gold seed solution. 
Table S1. pH, diameter and polydispersity index (PDI) of PAH-Au(III) solutions.

\begin{tabular}{lccc}
\hline & pH & $\begin{array}{c}\text { Diameter } \\
\text { nm }\end{array}$ & PDI \\
\hline $\boldsymbol{P A H - A u ( I I I )}$ & 2.5 & 26 & 0.28 \\
$\boldsymbol{P A H - A u ( I I I )}$ & 4.1 & 118 & 0.31 \\
$\boldsymbol{P A H - A u ( I I I )}$ & 4.8 & 143 & 0.32 \\
\hline Data represent the mean of 5 consecutive runs at $22^{\circ} \mathrm{C}$.
\end{tabular}

\section{References}

[1] L. Scarabelli, A. Sánchez-Iglesias, J. Pérez-Juste, L. M. Liz-Marzán, J. Phys. Chem. Lett. 2015, 6, 4270.

[2] B. G. Mathapa, V. N. Paunov, Soft Matter 2013, 9, 4780.

[3] Z. Cao, P. I. Gordiichuk, K. Loos, E. J. R. Sudhölter, L. C. P. M. De Smet, Soft Matter 2016, 12, 1496.

[4] B. L. Rivas, G. V. Seguel, Polyhedron 1999, 18, 2511.

[5] J. J. Harris, P. M. DeRose, M. L. Bruening, J. Am. Chem. Soc. 1999, 121, 1978. 
